\title{
A simple proposal for the publication of journal citation distributions
}

\author{
Vincent Larivière ${ }^{1}$, Véronique Kiermer ${ }^{2}$, Catriona J. MacCallum ${ }^{3}$, Marcia McNutt ${ }^{4 \dagger}$, Mark Patterson ${ }^{5}$, \\ Bernd Pulverer ${ }^{6}$, Sowmya Swaminathan ${ }^{7}$, Stuart Taylor ${ }^{8}$, Stephen Curry ${ }^{9 *}$
}

\begin{abstract}
${ }^{1}$ Associate Professor of Information Science, École de bibliothéconomie et des sciences de l'information, Université de Montréal, C.P. 6128, Succ. Centre-Ville, Montréal, QC. H3C 3J7, Canada; Observatoire des Sciences et des Technologies (OST), Centre Interuniversitaire de Recherche sur la Science et la Technologie (CIRST), Université du Québec à Montréal, CP 8888, Succ. Centre-Ville, Montréal, QC. H3C 3P8, Canada

${ }^{2}$ Executive Editor, PLOS, 1160 Battery Street, San Francisco, CA 94111, USA

${ }^{3}$ Advocacy Director, PLOS, Carlyle House, Carlyle Road, Cambridge CB4 3DN, UK

${ }^{4}$ Editor-in-Chief, Science journals, American Association for the Advancement of Science, 1200 New York Avenue, NW, Washington, DC 20005, USA

${ }^{5}$ Executive Director, eLife, 24 Hills Road, Cambridge CB2 1JP, UK

${ }^{6}$ Chief Editor, The EMBO Journal, Meyerhofstrasse 1,69117 Heidelberg, Germany

${ }^{7}$ Head of Editorial Policy, Nature Research, Springer Nature, 225 Bush Street, Suite 1850, San Francisco 94104, USA

${ }^{8}$ Publishing Director, The Royal Society, 6-9 Carlton House Terrace, London SW1Y 5AG, UK

9Professor of Structural Biology, Department of Life Sciences, Imperial College, Exhibition Road, London, SW7 2AZ, UK
\end{abstract}

†Present address: National Academy of Sciences, 2101 Constitution Avenue NW, Washington, DC 20418, USA

*Corresponding Author. Email: s.curry@imperial.ac.uk

Copyright: ๑ 2016 The Authors. This is an open access article distributed under the terms of the CC-BY 4.0 License, which permits unrestricted use, distribution, and reproduction in any medium, provided the original authors and source are credited.

Competing interests: The authors have declared that no competing interests exist.

Funding: The authors have no funding or support to report.

\begin{abstract}
Although the Journal Impact Factor (JIF) is widely acknowledged to be a poor indicator of the quality of individual papers, it is used routinely to evaluate research and researchers. Here, we present a simple method for generating the citation distributions that underlie JIFs. Application of this straightforward protocol reveals the full extent of the skew of distributions and variation in citations received by published papers that is characteristic of all scientific journals. Although there are differences among journals across the spectrum of JIFs, the citation distributions overlap extensively, demonstrating that the citation performance of individual papers cannot be inferred from the JIF. We propose that this methodology be adopted by all journals as a move to greater transparency, one that should help to refocus attention on individual pieces of work and counter the inappropriate usage of JIFs during the process of research assessment.
\end{abstract}

\section{Introduction}

The problem of over-reliance on the Journal Impact Factor (JIF) ${ }^{1}$ for research and researcher assessment has grown markedly in the 40 years since it emerged in 1972, conceived originally as a tool for librarians in making decisions on the purchase of journal subscriptions (1). Many stakeholders in academia and academic publishing have recognized that JIFs exert an undue influence in judgements made about individual researchers and individual research papers (2-5).

The main deficiencies of the JIF have been discussed in detail elsewhere $(2,3,6,7)$ but may be summarized as follows: the JIF is calculated inappropriately as the arithmetic mean of a highly skewed distribution of citations ${ }^{2}$; it contains no measure of the spread of the distribution; it obscures the high degree of overlap between the citation distributions of most journals; it is not reproducible and the data that support it are not publicly available $(8,9)$; it is quoted to a higher level of precision (three decimal places) than is warranted by the underlying data; it is based on a narrow two-year time window that is inappropriate for many disciplines and takes no account of the large variation in citation levels across disciplines (10); it includes citations to 'non-citable' items and citations to primary research paper are conflated with citations to reviews - it is therefore open to gaming and subject to negotiation with Thomson Reuters $(7,11,12)$; its relationship with citations received by individual papers is questionable and weakening (13).

We welcome the efforts of others to highlight the perturbing effects of JIFs on research assessment (notably, the San Francisco Declaration on Research Assessment (DORA) (14), the Leiden Manifesto (15), the Metric Tide report (16)) and their calls for concrete steps to mitigate their influence. We also applaud public statements by funders around the world (e.g. Research Councils UK (17), the Wellcome Trust (18), the European Molecular Biology Organisation (EMBO) (19), the Australian Research Council (20), and the Canadian Institutes of Health Research (21)) that no account should be taken of JIFs in assessing grant applications. And we are encouraged by those journals that have cautioned against the misappropriation of JIFs in researcher assessment (7, 11, 22-25).

But at the same time we recognize that many academics and many institutions lack confidence in the ability of the members of funding, promotion or other research assessment panels to shed what has become for many a habit of mind. This is exacerbated by the fact that various indicators are increasingly part of the toolbox of research management (16) and are often viewed as a convenient proxy for 'quality' by busy academics perennially faced with sifting large numbers of grant applications or CVs.

To challenge the over-simplistic interpretation of JIFs, we present here a simple methodology for generating the citation distribution of

\footnotetext{
${ }^{1}$ The JIF is formally defined as the mean number of citations received in a given year by papers published in a journal over the two previous years.

2 Although the JIF is presented as an arithmetic mean, the numerator is the total number of citations received by all documents published in the journal whereas the denominator is the subset of documents that Thomson Reuters classifies as 'citable' (i.e. 'Articles' and 'Reviews').
} 
Larivière et al. (2016) - Publication of Journal Citations

Table 1. Citations received in 2015 by document type published in 2013 and 2014

\begin{tabular}{|c|c|c|c|c|c|c|c|c|c|c|c|c|}
\hline \multirow[t]{2}{*}{ Journal } & \multicolumn{2}{|c|}{ Article } & \multicolumn{2}{|c|}{ Review } & \multicolumn{2}{|c|}{ Correction } & $\begin{array}{l}\text { Editorial- } \\
\text { Material }\end{array}$ & \multicolumn{2}{|c|}{$\begin{array}{c}\text { Others } \\
\text { documents }\end{array}$} & \multicolumn{2}{|c|}{$\begin{array}{l}\text { Unmatched } \\
\text { Citations }\end{array}$} & \multirow{2}{*}{$\begin{array}{r}\text { Total } \\
\text { Citations }\end{array}$} \\
\hline & $\mathrm{N}$. & $\%$ & $\mathrm{~N}$. & $\%$ & $\mathrm{~N}$. & $\%$ & N. $\quad \%$ & $\mathrm{~N}$. & $\%$ & $\mathrm{~N}$. & $\%$ & \\
\hline eLife & 5,459 & $84.4 \%$ & & & 10 & $0.2 \%$ & $98 \quad 1.5 \%$ & & & 902 & $13.9 \%$ & 6,469 \\
\hline EMBO J. & 3,219 & $82.2 \%$ & 472 & $12.1 \%$ & 2 & $0.1 \%$ & $1213.1 \%$ & 4 & $0.1 \%$ & 97 & $2.5 \%$ & 3,915 \\
\hline J. Informetrics & 387 & $92.6 \%$ & 6 & $1.4 \%$ & 1 & $0.2 \%$ & & 10 & $2.4 \%$ & 14 & $3.3 \%$ & 418 \\
\hline Nature & 54,143 & $83.2 \%$ & 3,554 & $5.5 \%$ & 47 & $0.1 \%$ & $2,770 \quad 4.3 \%$ & 1,681 & $2.6 \%$ & 2,903 & $4.5 \%$ & 65,098 \\
\hline Nature Comm. & 43,957 & $88.5 \%$ & 82 & $0.2 \%$ & 15 & $0.0 \%$ & & & & 5,609 & $11.3 \%$ & 49,663 \\
\hline PLOS Biol. & 2,927 & $87.0 \%$ & 16 & $0.5 \%$ & & & $2016.0 \%$ & & & 219 & $6.5 \%$ & 3,363 \\
\hline PLOS Genet. & 9,964 & $91.6 \%$ & 238 & $2.2 \%$ & 3 & $0.0 \%$ & $46 \quad 0.4 \%$ & & & 621 & $5.7 \%$ & 10,872 \\
\hline PLOS ONE & 168,590 & $90.7 \%$ & 2,753 & $1.5 \%$ & 86 & $0.0 \%$ & $50.0 \%$ & & & 14,378 & $7.7 \%$ & 185,812 \\
\hline Proc. R. Soc. B & 4,462 & $76.3 \%$ & 436 & $7.5 \%$ & 4 & $0.1 \%$ & $310.5 \%$ & & & 916 & $15.7 \%$ & 5,849 \\
\hline Science & 43,665 & $75.6 \%$ & 5,816 & $10.1 \%$ & 4 & $0.0 \%$ & $4,522 \quad 7.8 \%$ & 1,011 & $1.8 \%$ & 2,747 & $4.8 \%$ & 57,765 \\
\hline Sci. Rep. & 29,668 & $86.2 \%$ & 1 & $0.0 \%$ & 11 & $0.0 \%$ & $20.0 \%$ & & & 4,750 & $13.8 \%$ & 34,432 \\
\hline
\end{tabular}

papers published in any journal. Consistent with previous analyses $(9,26)$, application of this method to a selection of journals covering a number of different scientific disciplines shows that their citation distributions are skewed such that most papers have fewer citations than indicated by the JIF and, crucially, that the spread of citations per paper typically spans two to three orders of magnitude resulting in a great deal of overlap in the distributions for different journals. Although these features of citation distributions are well known to bibliometricians and journal editors $(7,23,26)$, they are not widely appreciated in the research community. It is the desire to broaden this awareness that motivated us, a group drawn from the research, bibliometrics and journals communities, to conduct the analysis reported here.

We believe that the wider publication of citation distributions provides a healthy check on the misuse of JIFs by focusing attention on their spread and variation, rather than on single numbers that conceal these universal features and assume for themselves unwarranted precision and significance. We propose that this methodology be adopted by all journals that publish their impact factors so that authors and readers are provided with a clearer picture of the underlying data. This proposal echoes the reasonable requests that journal reviewers and editors make of authors to show their data in justifying the claims made in their papers.

\section{Methods}

Purchased Database Method: The analyses presented here were conducted using the three main citation indexes purchased from Thomson Reuters by the Observatoire des sciences et des technologies (OST-UQAM): the Science Citation Index Expanded, the Social Science Citation Index, and the Arts and Humanities Citation index. Data were obtained on March $18^{\text {th }} 2016$ and the results reflect the content of the database at that point in time. They may therefore differ from results obtained subsequently using its Web version, the Web of Science ${ }^{\mathrm{TM}}$, which is continuously updated (see below), though any differences are likely to be small for distributions calculated over equivalent time windows.

To obtain the number of citations per citable item (which we defined as articles and reviews, following Thomson Reuters practice in JIF calculations (27)), we used Thomson Reuters' matching key to define links between citing and cited papers. As part of our analysis, additional citations were retrieved from the database using the various forms of each journal's name ${ }^{3}$. Although these could not be linked to specific papers and cannot therefore be included in the citation distributions, they are listed as unmatched citations in Table 1 to give an idea of the numbers involved. It is worth noting that these unmatched citations are included in the calculation of the JIF. For the journals eLife, Scientific Reports, Proceedings of the Royal Society B: Biology Sciences, and Nature Communications, the share of unmatched citations is higher, which suggests that citations to specific papers are underestimated by the Thomson Reuters matching key (Table 1). Thus, these distributions underestimate the numbers of citations per paper - and may overestimate the numbers of papers with zero citations. Given that these unmatched citations are likely to be evenly distributed across all papers, this effect should not affect the structure of the distributions.

Subscription Database Method: The use of a purchased database provides convenient access the bulk citation data, but the expense involved means the method described above is only likely to be a viable option for professional bibliometricians. To facilitate the generation of citation distributions by non-specialists, we developed step-by-step protocols that rely on access to essentially the same data via subscription to either the Web of Science ${ }^{\mathrm{TM}}$ (Thomson Reuters Inc.) or Scopus ${ }^{\mathrm{TM}}$ (Elsevier BV). The details of each protocol are presented in Appendices 1 and $2^{4}$.

It should be noted that all the protocols we present here for generating distributions use only those citations that are unambiguously matched to specific papers. This is in contrast to the approach used by Thomson Reuters in calculating JIFs which includes citations to all document types as well as unmatched citations (see Table 1). Thus, while the cohort of articles can be matched to the JIF cohort (namely, citations received in 2015 to articles published in 2013 and 2014) the absolute values of the citations to individual articles and the total number of citations can vary substantially from that used in the JIF calculation.

\section{Results}

Using the Purchased Database Method described above, we generated frequency plots - or citation distributions - for 11 journals: eLife, EMBO Journal, Journal of Informetrics, Nature, Nature Communications, PLOS Biology, PLOS Genetics, PLOS ONE, Proceedings of the Royal Society B: Biology Sciences, Science and Scientific Reports (Figure 1). The journals selected are both multidisciplinary and subject-specific in scope, and range in impact factor from less than 3 to more than 30 . They represent journals from seven publishers: eLife Sciences, Elsevier, EMBO Press, Springer Nature, the Public Library of Science (PLOS), The Royal Society and the American Association for the Advancement of Science (AAAS).

In an attempt to relate our analyses to the widely-available JIFs for 2015, the period over which the citations accumulated for our

${ }^{3}$ For example, the journal Proceedings of the Royal Society $B$ - Biological Sciences appeared in the reference list as $P R$ SOC B, P R SOC B IN PRESS, PR SOC BIOL SCI, PR SOC LONDON B, etc.

${ }_{4}$ Since there are more journals and papers indexed in Scopus ${ }^{\mathrm{TM}}$, citation rates for individual articles are likely to be higher than those presented here if this database is used to generate distributions. 

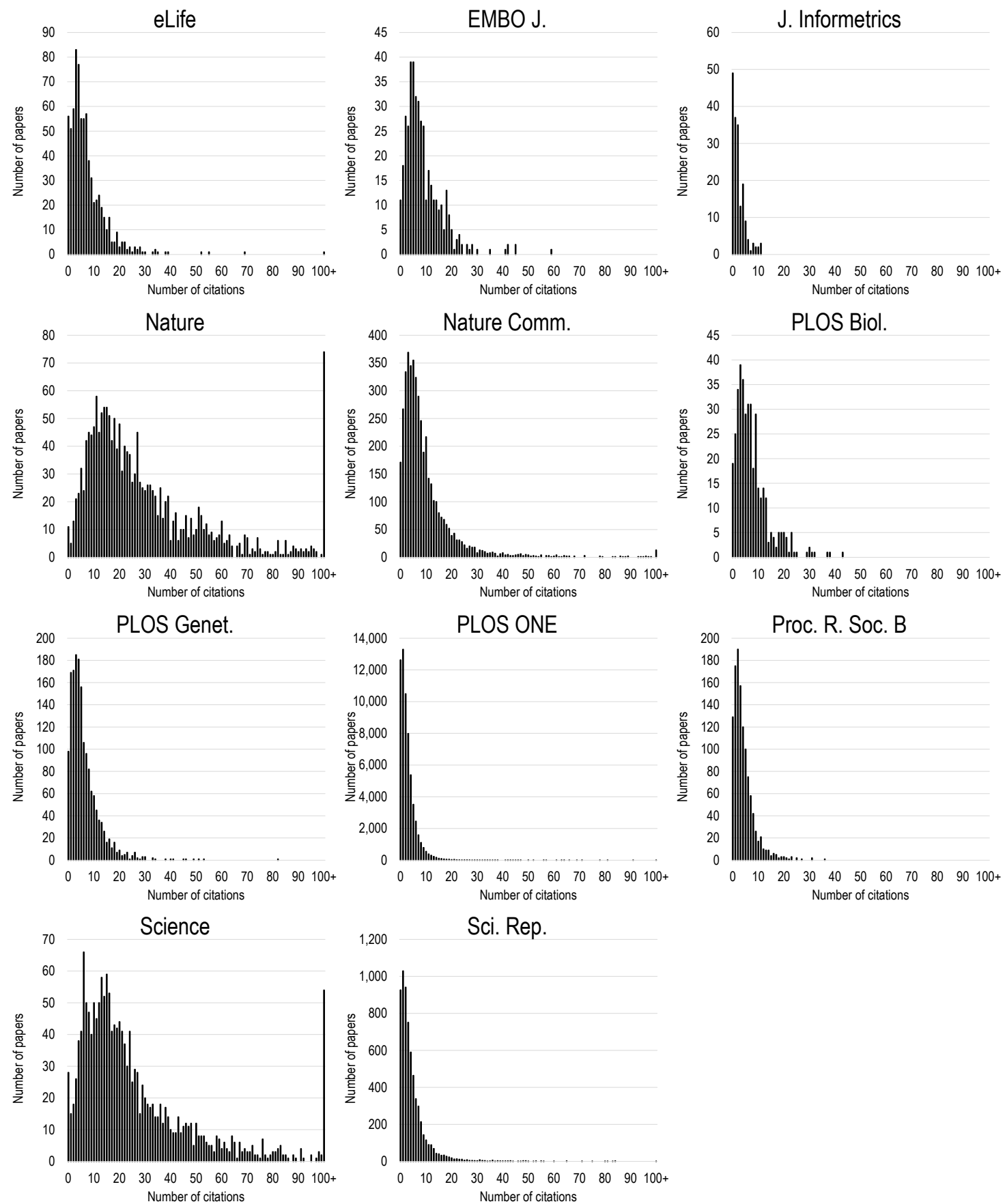

Fig 1. Citation distributions of 11 different science journals. Citations are to 'citable documents' as classified by Thomson Reuters, which include standard research articles and reviews. The distributions contain citations accumulated in 2015 to citable documents published in 2013 and 2014 in order to be comparable to the $2015 \mathrm{JIFs}$ published by Thomson Reuters. To facilitate direct comparison, distributions are plotted with the same range of citations (0-100) in each plot; articles with more than 100 citations are shown as a single bar at the right of each plot.

distributions was chosen to match that of the 2015 Journal Impact Factors published by Thomson Reuters - namely, the number of citations accrued in 2015 from documents published in 2013-2014. However, to more effectively compare journal distributions, we opted to include only citable items as classified by Thomson Reuters, which includes standard research articles and review articles (27), because different journals publish different amounts of additional content such as editorials, news items, correspondence, and commentary. It should also be noted that the definition of research and review articles used by Thomson Reuters does not always match the labels given to different document types by journals. Table 1 provides a summary of the number and percentage of articles and citations accrued for each document type within each journal as classified by Thomson Reuters. The summary data used to generate the distributions are provided in Supplemental File 1.

While the distributions presented in Figure 1 were generated using purchased data (see Methods), we tested whether similar distributions could be produced following the step-by-step Subscription Based Method outlined in Appendix 1 which uses data accessed online via Web of Science ${ }^{\mathrm{TM}}$. As seen in the distributions calculated for the EMBO Journal (Figure 2), the broad features of the distributions from these different sources are essentially identical, with differences being due to updates made on the database between purchase of data and time of online access. 


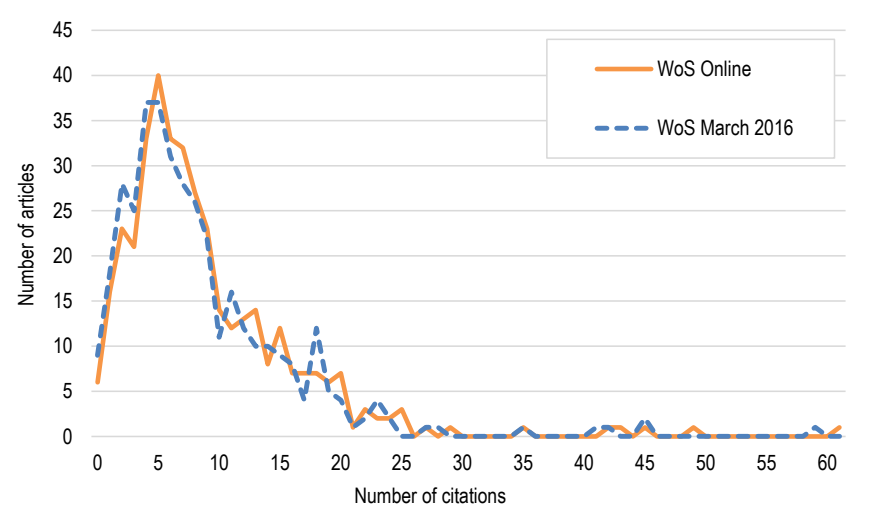

Fig 2. Comparison plot for EMBO Journal. The analyses in this paper are based on proprietary data bought from Thomson Reuters by the Observatoire des sciences et des technologies (OST-UQAM) and is similar to that used by Thomson Reuters to generate the JIFs ('WoS March 2016'). Publishers and Institutions with a subscription to the Web of Science ${ }^{\mathrm{TM}}$ have access to a different dataset ('WoS online').

For all journals, the shape of the distribution is highly skewed to the left, being dominated by papers with lower numbers of citations. Typically, $65-75 \%$ of the articles have fewer citations than indicated by the JIF (Table 2). The distributions are also characterized by long rightward tails; for the set of journals analyzed here, only $15-25 \%$ of the articles account for $50 \%$ of the citations as shown in the cumulative distributions plotted in Figure 3. The distributions are also broad, often spanning two or more orders of magnitude. The spread tends to be broader for journals with higher impact factors. Our results also show that journals with very high Impact Factors tend to have fewer articles with low numbers of citations.

The journals with highest impact factors (Nature and Science) also tend to have more articles with very high levels of citation within the two-year time period used for JIF calculations (and our analyses). The most cited articles in Nature and Science are cited 905 times and 694 times respectively in 2015 (see Supplemental File 1). Highly cited articles also appear in journals with much lower impact factors; for example, the most-cited articles in PLOS ONE and Scientific Reports are cited 114 and 141 times in 2015, respectively. For all journals, the very highly cited articles represent a small percentage of the total number of articles and yet have a disproportionate influence on the impact factor because it is based on an arithmetic mean calculation that does not take proper account of the skew in the distribution.

Despite the variations in citation distributions between journals that are evident in Figure 1, there is substantial overlap in the citation distributions across all the journals (Figure 4a). The overlap becomes more apparent when the number of articles are converted to percentages (Figure $4 \mathrm{~b}$ ). This makes it clear that, even without taking into account the effect of the sizes of different disciplines on citation counts, papers with high and low numbers of citations appear in most, if not all, journals.

Table 2: Percentage of papers published in 2013-2014 with number of citations below the value of the $2015 \mathrm{JIF}$.

\begin{tabular}{lrr}
\hline Journal & JIF & $\begin{array}{r}\% \text { citable items } \\
\text { below JIF }\end{array}$ \\
\hline eLife & 8.3 & $71.2 \%$ \\
EMBO J. & 9.6 & $66.9 \%$ \\
J. Informetrics & 2.4 & $68.4 \%$ \\
Nature & 38.1 & $74.8 \%$ \\
Nature Comm. & 11.3 & $74.1 \%$ \\
PLOS Biol. & 8.7 & $66.8 \%$ \\
PLOS Genet. & 6.7 & $65.3 \%$ \\
PLOS ONE & 3.1 & $72.2 \%$ \\
Proc. R. Soc. B & 4.8 & $65.7 \%$ \\
Science & 34.7 & $75.5 \%$ \\
Sci. Rep. & 5.2 & $73.2 \%$ \\
\hline
\end{tabular}

\section{Discussion}

The aim of this paper is to increase awareness of the journal citation distributions underlying JIFs by disseminating a simple protocol that allows them to be generated by anyone with access, via institutional or publisher subscription, to Web of Science ${ }^{\mathrm{TM}}$ or Scopus ${ }^{\mathrm{TM}}$ (Appendices 1 and 2). We have selected a group of journals for illustrative purposes and have made no attempt to be comprehensive. Our intention here is to encourage publishers, journal editors and academics to generate and publish journal citation distributions as a countermeasure to the tendency to rely unduly and inappropriately on JIFs in the assessment of research and researchers.

The proposed method is straightforward and robust. It generates citation distributions that have all the same features that have been identified in previous analyses $(9,26)$. The distributions reveal that

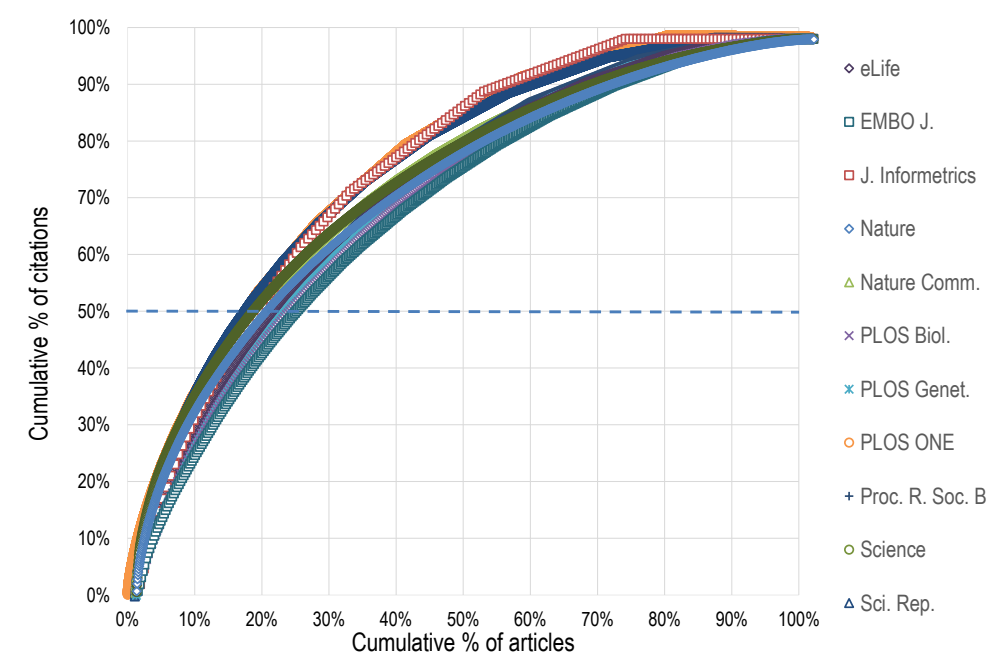

Fig 3. The cumulative $\%$ of citations and articles plotted for the 11 journals included in this study. The plots for all the journals are very similar, which reflects the skewness of the distributions shown in Figure 1. 
a

\section{0,000}

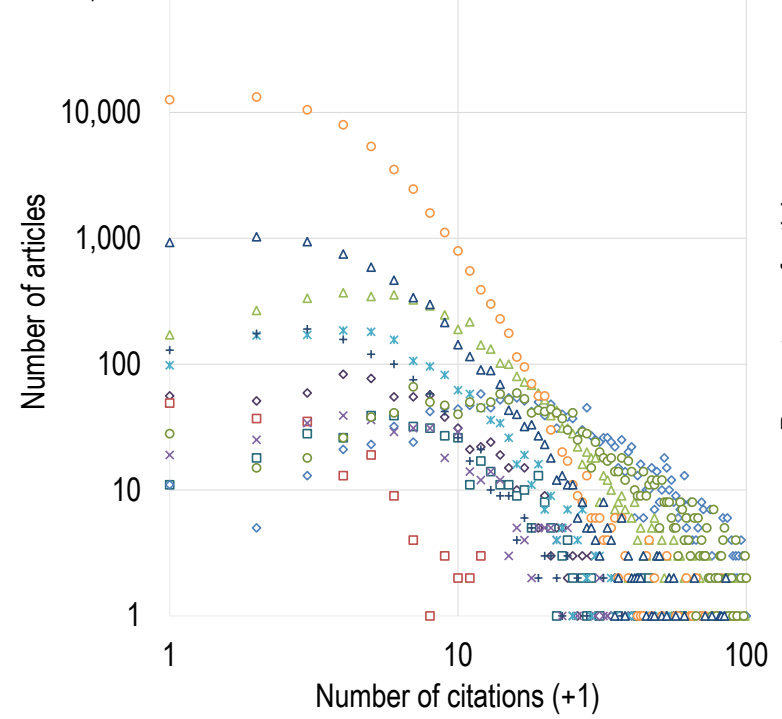

b

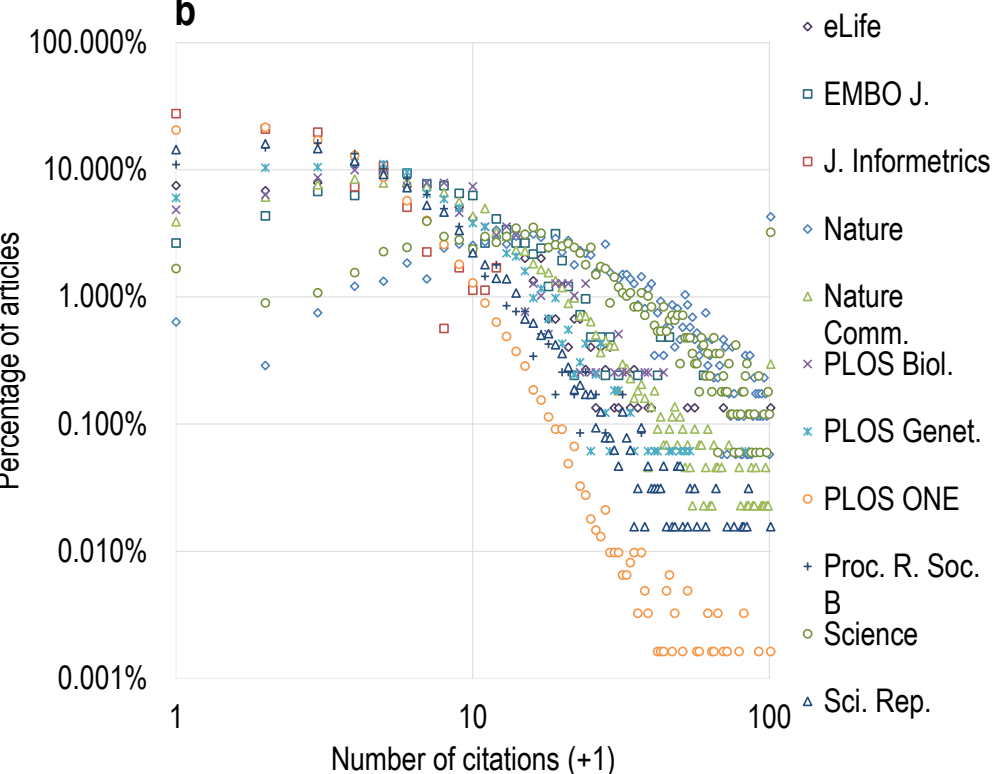

Number of citations (+1)

Fig 4. A log-scale comparison of the 11 citation distributions. (a) The absolute number of articles plotted against the number of citations. (b) The percentage of articles plotted against the number of citations.

for all journals, a substantial majority of papers have many fewer citations than indicated by the arithmetic mean calculation used to generate the JIF and that for many journals the spread of citations per paper varies by more than two orders of magnitude. Although JIFs do vary from journal to journal, the most important observation as far as research assessment is concerned, and one brought to the fore by this type of analysis, is that there is extensive overlap in the distributions for different journals. Thus for all journals there are large numbers of papers with few citations and relatively few papers with many citations.

This underscores the need to examine each paper on its own merits and serves as a caution against over-simplistic interpretations of the JIF. Users of JIFs should also appreciate other complicating factors, such as the inflationary effect on citations in journals with higher JIFs, which may be due to greater visibility and perceived prestige of such journals (28-30). This effect is illustrated by analysis of citations to a medical "white paper" that was published in eight different journals in 2007 and showed that the number of citations that each publication received correlated strongly $\left(\mathrm{R}^{2}=0.91\right)$ with the JIF of the host journal across a range of JIF values from 2 to 53 (31).

With one exception (J. Informetrics), our analyses cover a collection of journals that are generally broad in scope, encompassing several different disciplines across the sciences. It may be that the breadth of the distributions are less marked in journals of narrower scope, although their JIFs are just as prone to outlier effects and overlapping distributions of citations have been observed in more specialized journals $(9,32)$.

Despite the overlap, there are evident differences in the average citation performance of different journals, and we are not arguing that the JIF has no value in the comparison of journals (the significance of which has been analyzed by Royle (9)). Rather we hope that this analysis helps to expose the exaggerated value attributed to the JIF and strengthens the contention that it is an inappropriate indicator for the evaluation of research or researchers. On a technical point, the many unmatched citations (i.e. citations not clearly linked to a specific article, Table 1) that were discovered in the data for eLife, Nature Communications, Proceedings of the Royal Society: Biology Sciences and Scientific Reports raises concerns about the general quality of the data provided by Thomson Reuters. Searches for citations to eLife papers, for example, have revealed that the data in the Web of Science ${ }^{\mathrm{TM}}$ are incomplete owing to technical problems that Thomson Reuters is currently working to resolve. We have not investigated whether similar problems affect journals outside the set used in our study and further work is warranted. However, the raw citation data used here are not publicly available but remain the property of Thomson Reuters. A logical step to facilitate scrutiny by independent researchers would therefore be for publishers to make the reference lists of their articles publicly available. Most publishers already provide these lists as part of the metadata they submit to the Crossref metadata database (33) and can easily permit Crossref to make them public, though relatively few have opted to do so. If all Publisher and Society members of Crossref (over 5,300 organisations) were to grant this permission, it would enable more open research into citations in particular and into scholarly communication in general (33).

The co-option of JIFs as a tool for assessing individual articles and their authors, a task for which they were never intended, is a deeply embedded problem within academia and one that has no easy solutions. We hope that by facilitating the generation and publication of journal citation distributions, the influence of the JIF in research assessment might be attenuated, and attention focused more readily onto the merits of individual papers - and onto the diverse other contributions that researchers make to research such as sharing data, code, and reagents (not to mention their broader contributions, such as peer review and mentoring students, to the mission of the academy).

To advance this agenda we therefore make the following recommendations:

- We encourage journal editors and publishers that advertise or display JIFs to publish their own distributions using the above method, ideally alongside statements of support for the view that JIFs have little value in the assessment of individuals or individual pieces of work (see this example at the Royal Society). Large publishers should be able to do this through subscriptions to Web of Science ${ }^{\mathrm{TM}}$ or Scopus ${ }^{\mathrm{TM}}$; smaller publishers may be able to ask their academic editors to generate the distributions for their journals.

- We encourage publishers to make their citation lists open via Crossref, so that citation data can be scrutinized and analyzed openly.

- We encourage all researchers to get an ORCID iD, a digital identifier that provides unambiguous links to 
published papers and facilitates the consideration of a

broader range of outputs in research assessment.

These recommendations represent small but feasible steps that should improve research assessment. This in turn should enhance the confidence of researchers in judgements made about them and, possibly, the confidence of the public in the judgements of researchers. This message is supported by the adoption in many journals of article-level metrics and other indicators that can help to track the use of research paper within and beyond the academy. We recognize that drawing attention to citation distributions risks inadvertent promotion of JIFs. However, we hope that the broader message is clear: research assessment needs to focus on papers rather than journals, keeping in mind that downloads and citation counts cannot be considered as reliable proxies of the quality of an individual piece of research (16). We would always recommend that a research paper is best judged by reading it.

\section{Acknowledgements}

We are very grateful to Valda Vinson and Monica Bradford for critical reading of the manuscript.

\section{Supplemental Files}

Supplemental File 1: Microsoft Excel spreadsheet containing the summary data used to prepare the Figures and Tables for this paper. Also contains the Figures and Tables themselves.

Supplemental File 2: Microsoft PowerPoint file containing readyto-use, high-resolution slides of the Figures and Tables from this paper.

Supplemental File 3: PDF version of Supp. File 2, containing ready-to-use, high-resolution slides of the Figures and Tables from this paper.

\section{Author Contributions Statement}

Vincent Larivière: methodology, formal analysis, investigation, writing - original draft preparation, visualization

Véronique Kiermer: writing - review and editing

Catriona MacCallum: writing - original draft preparation, review and editing

Marcia McNutt: writing - review and editing

Mark Patterson: writing - original draft preparation, review and editing

Bernd Pulverer: writing - review and editing

Sowmya Swaminathan; writing - review and editing

Stuart Taylor: methodology, formal analysis, investigation, writing - original draft preparation, visualization

Stephen Curry: conceptualization, investigation, writing - original draft preparation, review and editing

\section{References}

1. Garfield E. (1972) Citation Analysis as a Tool in Journal Evaluation Journals Can Be Ranked by Frequency and Impact of Citations for Science Policy Studies. Science. 178:471-9.

2. Seglen PO. (1997) Why the impact factor of journals should not be used for evaluating research. BMJ. 314:498-502.

3. Arrnold DN, Fowler KK. (2011) Nefarious numbers. Notices of the AMS 58:434-7.

4. Curry S. (2012) Sick of Impact Factors. Reciprocal Space. Available from: http://occamstypewriter.org/scurry/2012/08/13/sick-of-impactfactors/Accessed 15 June 2016.

5. Casadevall A, Fang FC. (2014) Causes for the persistence of impact factor mania. MBio. 5:e0064-14.

6. Archambault E, Lariviere V. (2009) History of the journal impact factor: Contingencies and consequences. Scientometrics. 79:635-49.

7. Pulverer B. (2015) Dora the Brave. EMBO J. 34:1601-2.
8. Rossner M, Van Epps H, Hill E. (2007) Show me the data. J Exp Med. 204:3052-3.

9. Royle S. (2015) Wrong Number: A Closer Look at Impact Factors. Quantixed. Available from: https://quantixed.wordpress.com/ 2015/05/05/wrong-number-a-closer-look-at-impact-factors/Accessed 15 June 2016.

10. Althouse BM, West JD, Bergstrom CT, Bergstrom T. (2009) Differences in Impact Factor Across Fields and Over Time. J Am Soc Inf Sci Tec. 60:27-34.

11. Editorial. (2006) The impact factor game. It is time to find a better way to assess the scientific literature. PLoS Med. 3:e291.

12. Martin BR. (2016) Editors' JIF-boosting stratagems - Which are appropriate and which not? Res Policy. 45:1-7.

13. Lozano GA, Lariviere V, Gingras Y. (2012) The weakening relationship between the impact factor and papers' citations in the digital age. $J \mathrm{Am}$ Soc Inf Sci Tec. 63:2140-5.

14. San Francisco Declaration on Research Assessment (DORA) Available at: http://www.ascb.org/dora/. Accessed 15 June 2016.

15. Hicks D, Wouters P, Waltman L, de Rijcke S, Rafols I. (2015) Bibliometrics: The Leiden Manifesto for research metrics. Nature. 520:429-31.

16. Wilsdon J, Allen E, Belfiore E, Campbell P, Curry S, Hill S, et al. (2015) The Metric Tide: Report of the Independent Review of the Role of Metrics in Research Assessment and Management. Available at: http:// www.hefce.ac.uk/pubs/rereports/Year/2015/metrictide/Title, 104463,en.html. Accessed 15 June 2015.

17. Research Councils UK. RCUK Policy on Open Access. Available at: http://www.rcuk.ac.uk/research/openaccess/policy/. Accessed 23 June 2016.

18. Wellcome Trust. Open Access Policy. Available at: https:// wellcome.ac.uk/funding/managing-grant/open-access-policy. Accessed 23 June 2016.

19. European Molecular Biology Organisation. (2016) EMBO Long-Term Fellowships: Guidelines for Applicants. Available at: http:// www.embo.org/documents/LTF/LTF_Guidelines for Applicants.pdf. Accessed 20 June 2015.

20. Austrialian Research Council. (2016) Assessor Handbook for Detailed Assessors: A guide for Detailed Assessors assessing Proposals for. Available at: http://www.arc.gov.au/sites/default/files/filedepot/Public/ NCGP/handbooks/fl16 round_1_detailed.pdf. Accessed 15 June 2015.

21. Canadian Institutes of Health Research. CIHR Peer Review Manual for Grant Applications. Available at: http://www.cihr-irsc.gc.ca/e/4656.html -s2_3. Accessed 23 June 2016.

22. Editorial. (2005) Not-so-deep impact. Nature. 435:1003-4.

23. Editorial. (2013) Beware the impact factor. Nat Mater. 12:89.

24. Alberts B. (2013) Impact factor distortions. Science. 340:787.

25. Schekman R, Patterson M. (2013) Reforming research assessment. eLife. 2: 00055 .

26. Seglen PO. (1992) The Skewness of Science. J Am Soc Inform Sci. 43:628-38.

27. Thomson Reuters. The Thomson Reuters Impact Factor Available at: http://wokinfo.com/essays/impact-factor/. Accessed 15 June 2016.

28. Davis P. (2010) Impact Factors - A Self-fulfilling Prophecy? The Scholarly Kitchen. Available from: https://scholarlykitchen.sspnet.org/ 2010/06/09/impact-factors-a-self-fulfilling-prophecy/ Accessed 15 June 2016.

29. Perneger TV. (2010) Citation analysis of identical consensus statements revealed journal-related bias. J Clin Epidemiol. 63:660-4.

30. Lariviere V, Gingras Y. (2010) The Impact Factor's Matthew Effect: A Natural Experiment in Bibliometrics. J Am Soc Inf Sci Tec. 61:424-7.

31. Cantrill S. (2016) Imperfect impact. Chemical Connections. Available from: https://stuartcantrill.com/2016/01/23/imperfect-impact/ Accessed 15 june 2016.

32. Cantrill S. (2015) Chemistry journal citation distributions. Chemical Connections. Available from: https://stuartcantrill.com/2015/12/10/ chemistry-journal-citation-distributions/Accessed 15 June 2016.

33. Bilder G. (2016) Distributing references via Crossref. The Art of Persistence. Available from: http://blog.crossref.org/2016/06/ distributing-references-via-crossref.html Accessed 23 June 2016. 
bioRxiv preprint doi: https://doi.org/10.1101/062109. The copyright holder for this preprint (which was not peer-reviewed) is the author/funder. It is made available under a CC-BY 4.0 International license.

Larivière et al. (2016) - Publication of Journal Citations

Appendix 1 - Method for generating the journal citation distribution graph from the Web of Science $^{\mathrm{TM}}$ (2014 Impact Factor set)

The example given below is for generating distributions over the two-year window (2012-2013) that is used in calculation of the 2014 Journal Impact Factor. For later years, such as for the distributions based on the 2015 JIF in the main article here, the two-year window should be adjusted accordingly.

1. In Web of Science, select Core Collection.

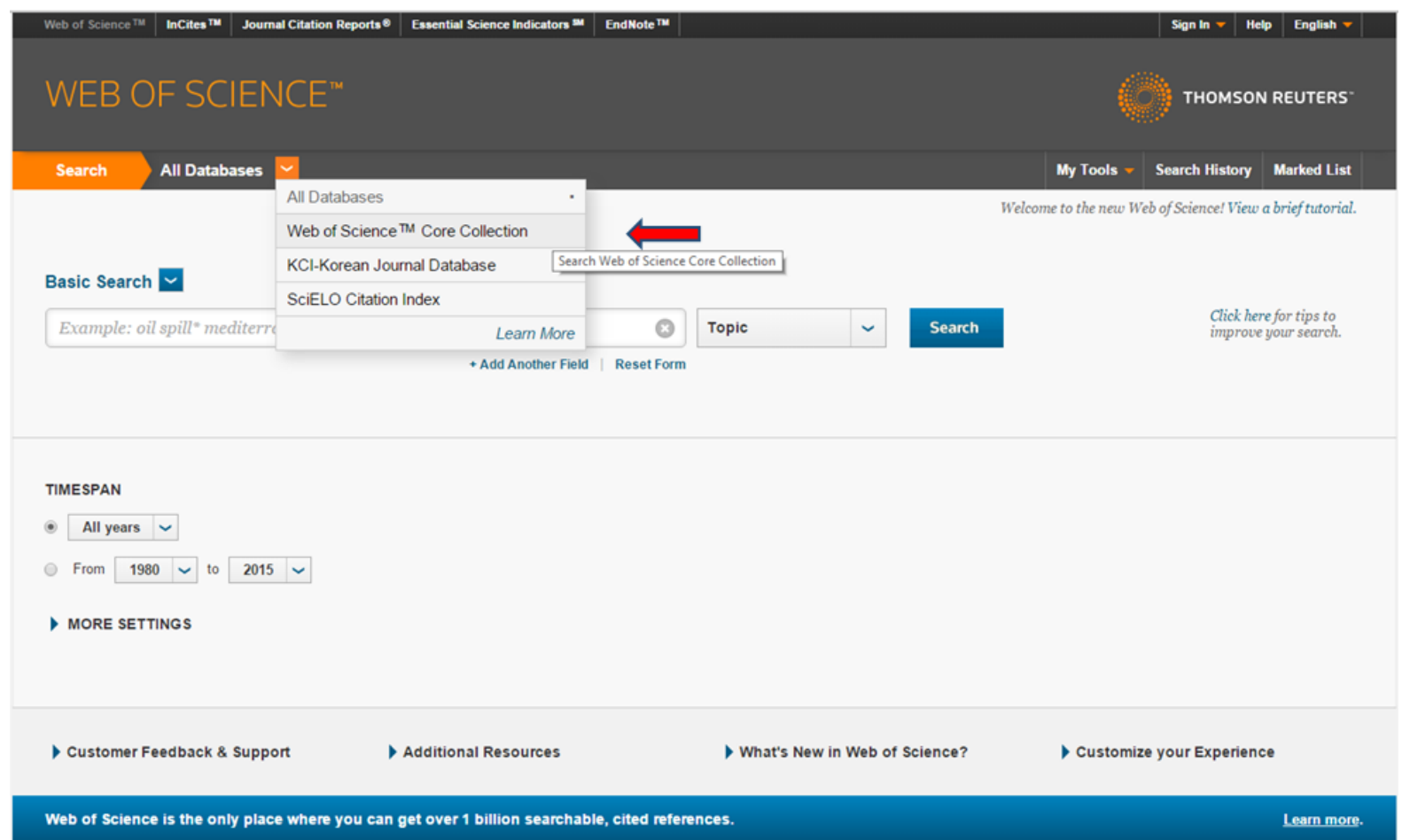


2. Select 'Publication Name' as the filter for the first field and then enter the journal name in the associated free text box. Select the 'Add Another Field' option and select 'Year Published' as the second filter and enter 2012-2013 in the text box. Click search. In the example shown, the journal Biology Letters has been selected.
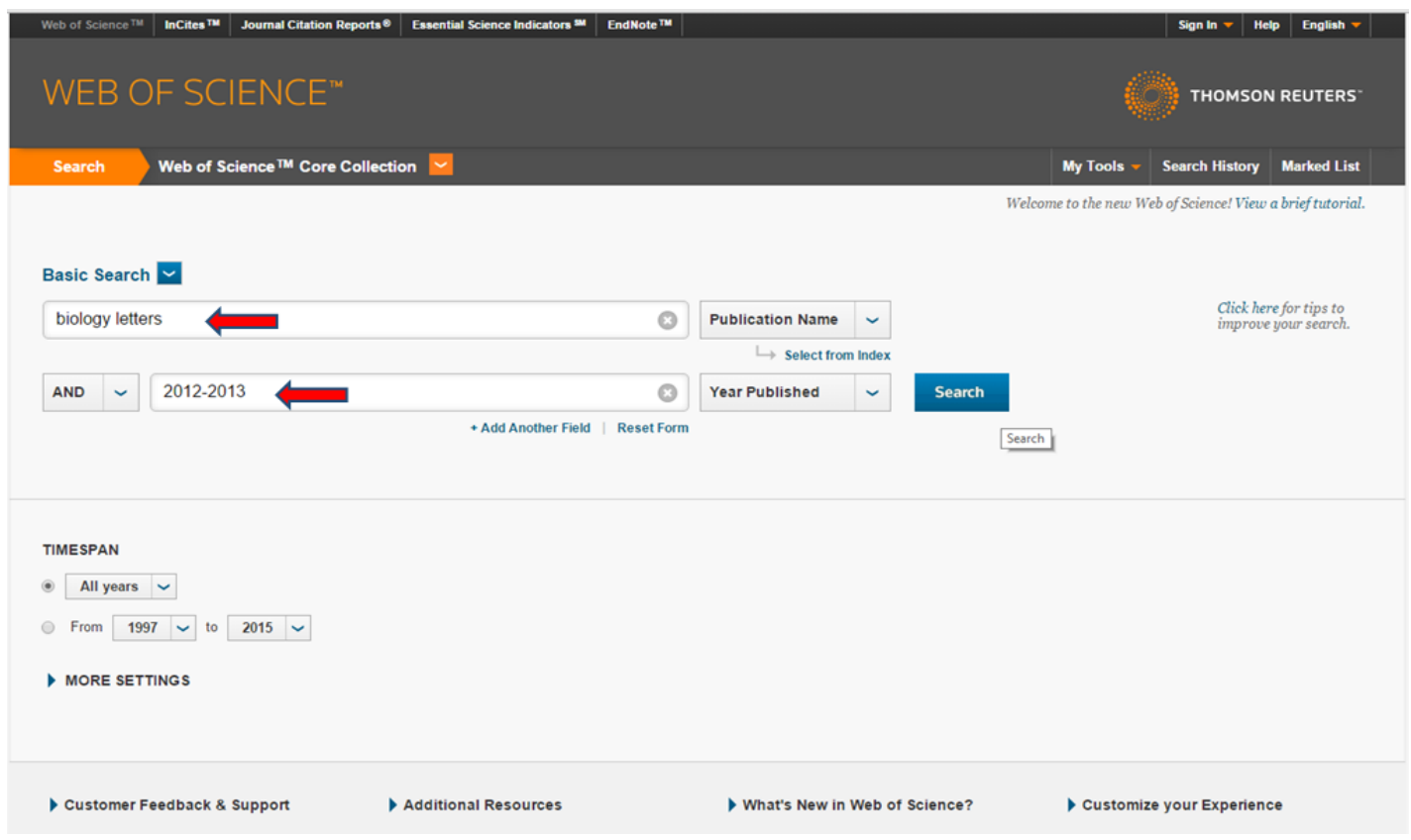

3. That produces the requisite article set. Next, click Create Citation Report. (To match as closely as possible the distributions shown in the analyses in this paper, limit the search to 'Articles' and 'Reviews' using the buttons on the left hand side of the screen under 'Document Types'.). Note that, as in the screenshot below, if the journal does not publish reviews (as classified by Thomson Reuters), an option to tick 'Reviews' will not be available.

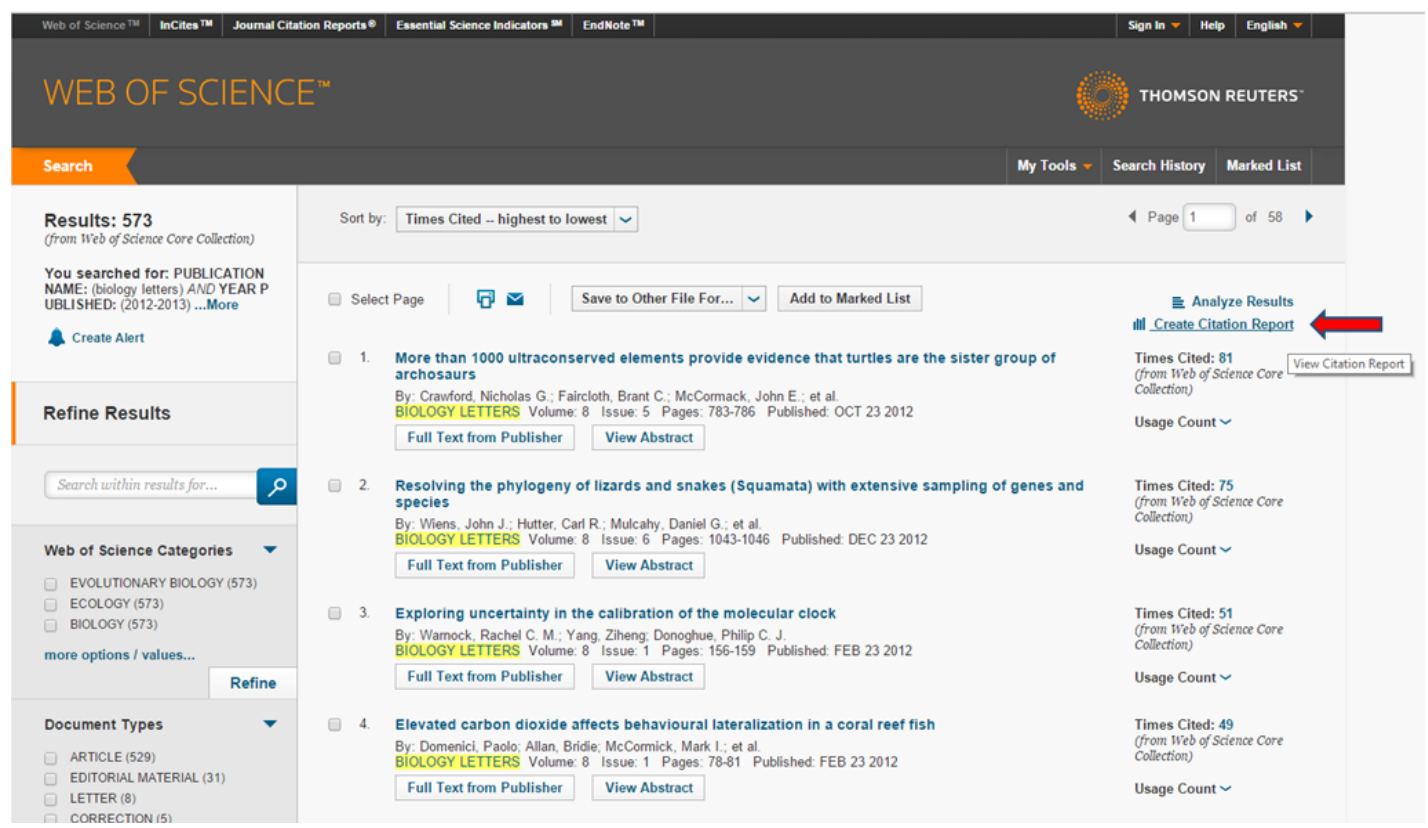


bioRxiv preprint doi: https://doi.org/10.1101/062109. The copyright holder for this preprint (which was not peer-reviewed) is the author/funder. It is made available under a CC-BY 4.0 International license.

Larivière et al. (2016) - Publication of Journal Citations

4. The citation report should look similar to this. Note the number of articles retrieved by the search at the top of the page (573 in example below).

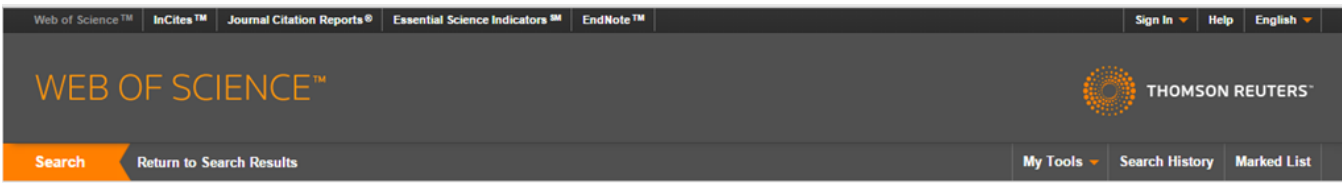

Citation Report: 573
(from Web of Science Core Colliction

You searched for. PUBLICATION NAME: (biology letters) AND YEAR PUBLISHED: (2012-2013) _...More

This report reffects citatons to source items indexed within Web of Science Core Collection. Perform a Cited Reference Search to include citasons to items not tindered within Web of Science Core Collection.
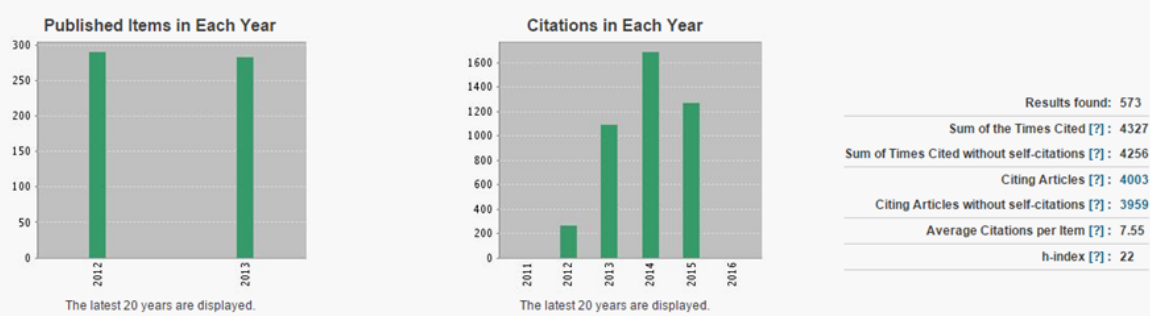

Sort by: Times Cited - highest to lowest

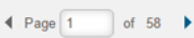

Use the checkboxes to remove individual items from this Citation Report

- or restrict to items published between $1997 \checkmark$ and $2016 \checkmark$ Co

More than 1000 ultraconserved elements provide evidence that turtles are the sister group of archosaurs

By: Crawford, Nicholas G; Faircloth, Brant C. McCormack, John E; et al
BIOLOGY LETTERS Volume: 8 Issue: 5 Pages: $783-786$ Published OCT 232012

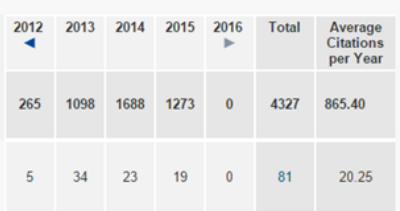

5. Scroll to the bottom of the web-page and export the list to Excel.

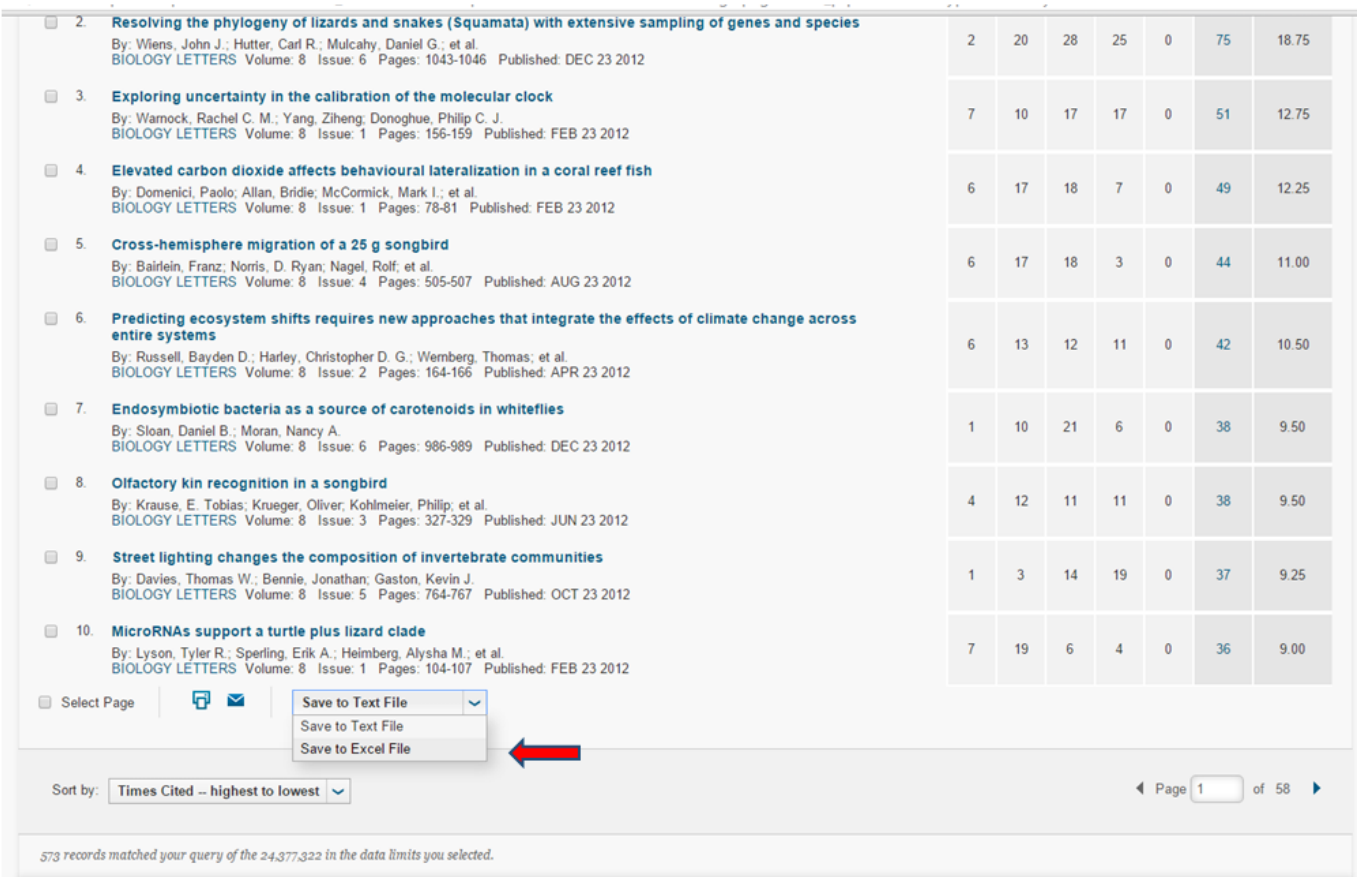


6. When prompted, enter the number of articles retrieved by the search as the maximum number of records. Web of Science ${ }^{\mathrm{TM}}$ will only process 500 records at a time, so if you have more articles than that, you'll need to export several Excel files and then combine them.

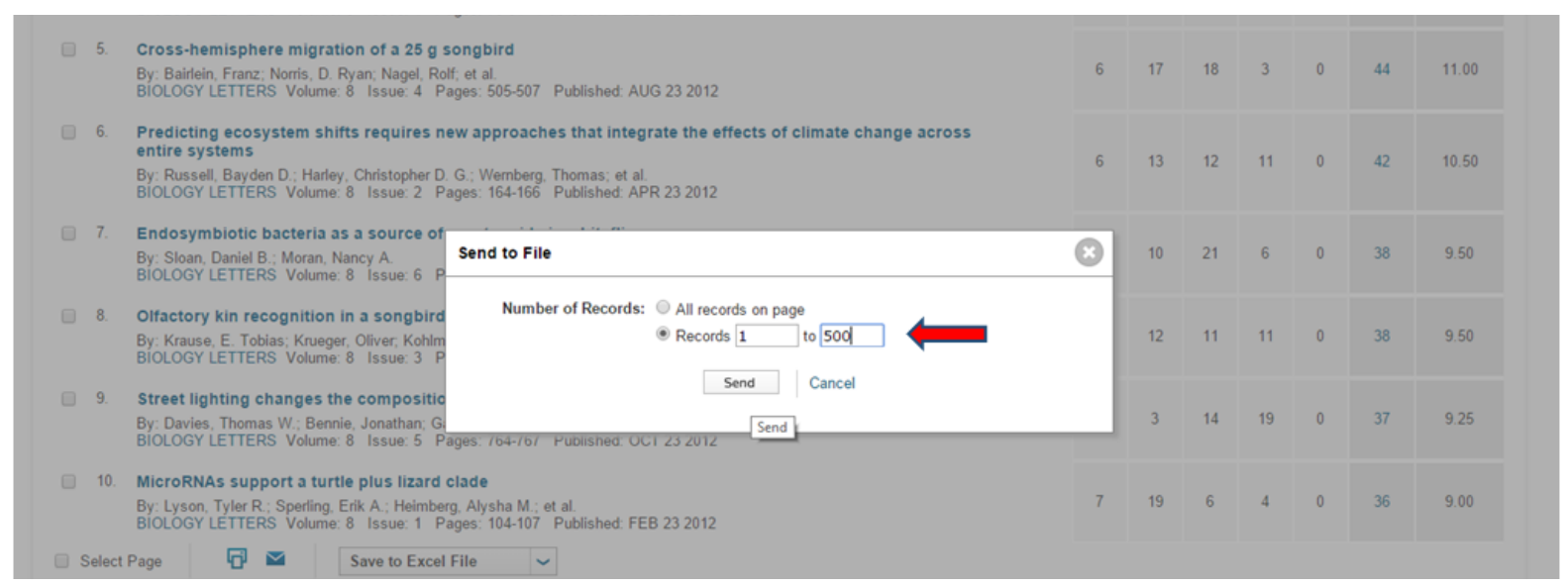

7. Open the combined file in Excel.

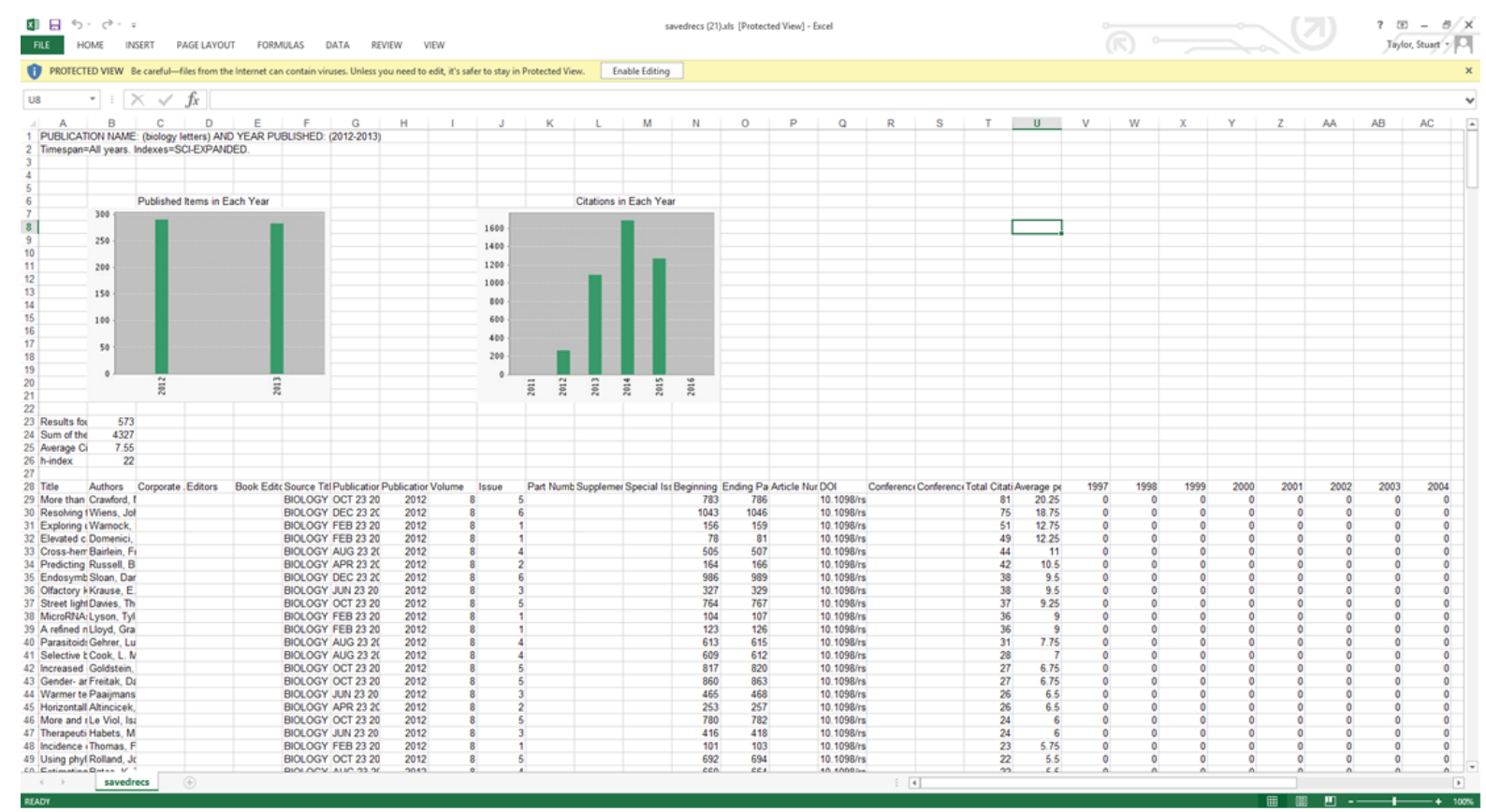


8. Only the column for the citations received in 2014 is needed for the distribution, so scroll across and select that column.

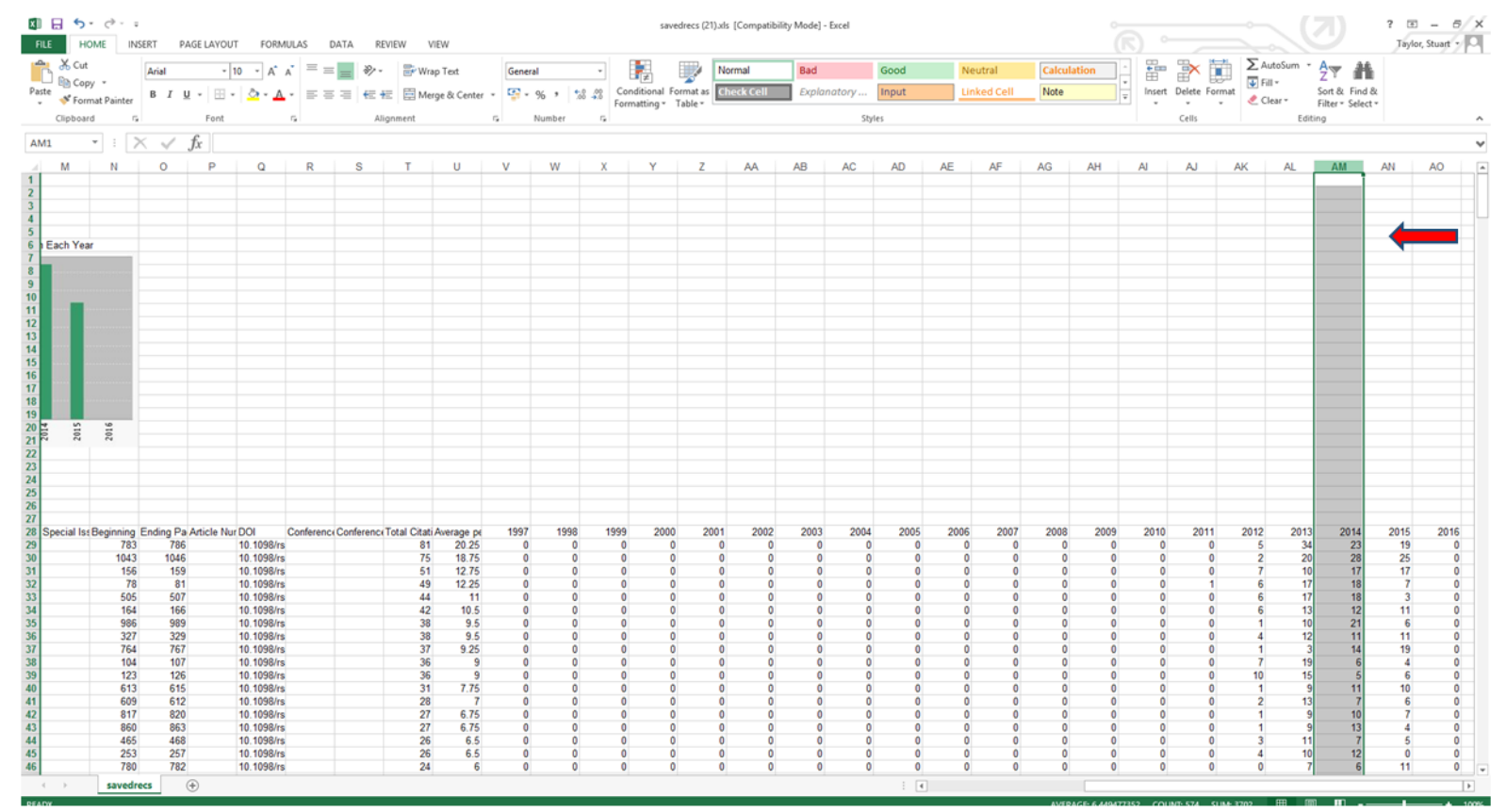

9. Sort the column into descending order (omitting the '2014' label at the top).

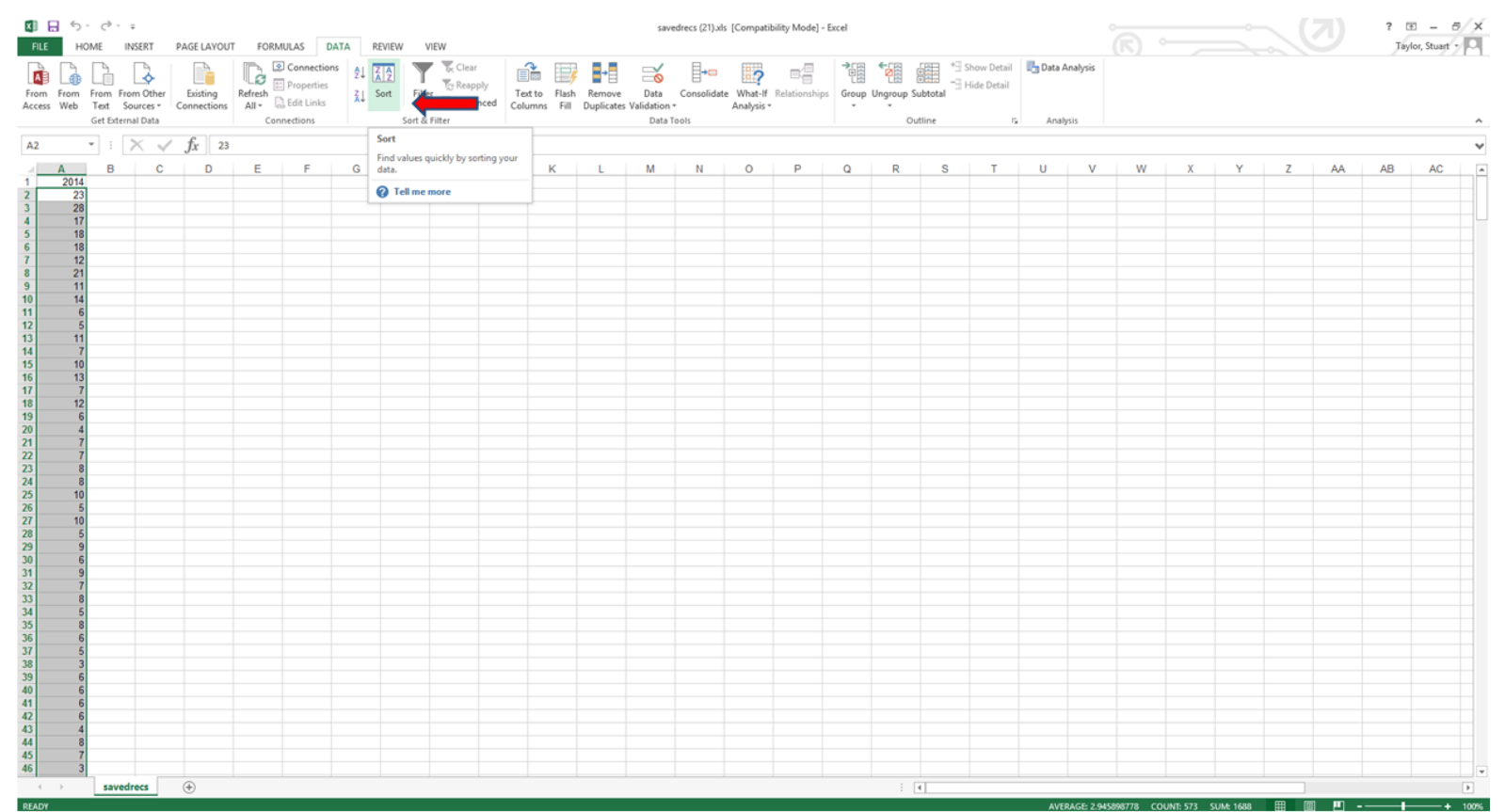


10. Note the maximum citation $(x)$ count and create a new column containing 0 to $X$ called "Citations". In the example shown below, $x=28$.

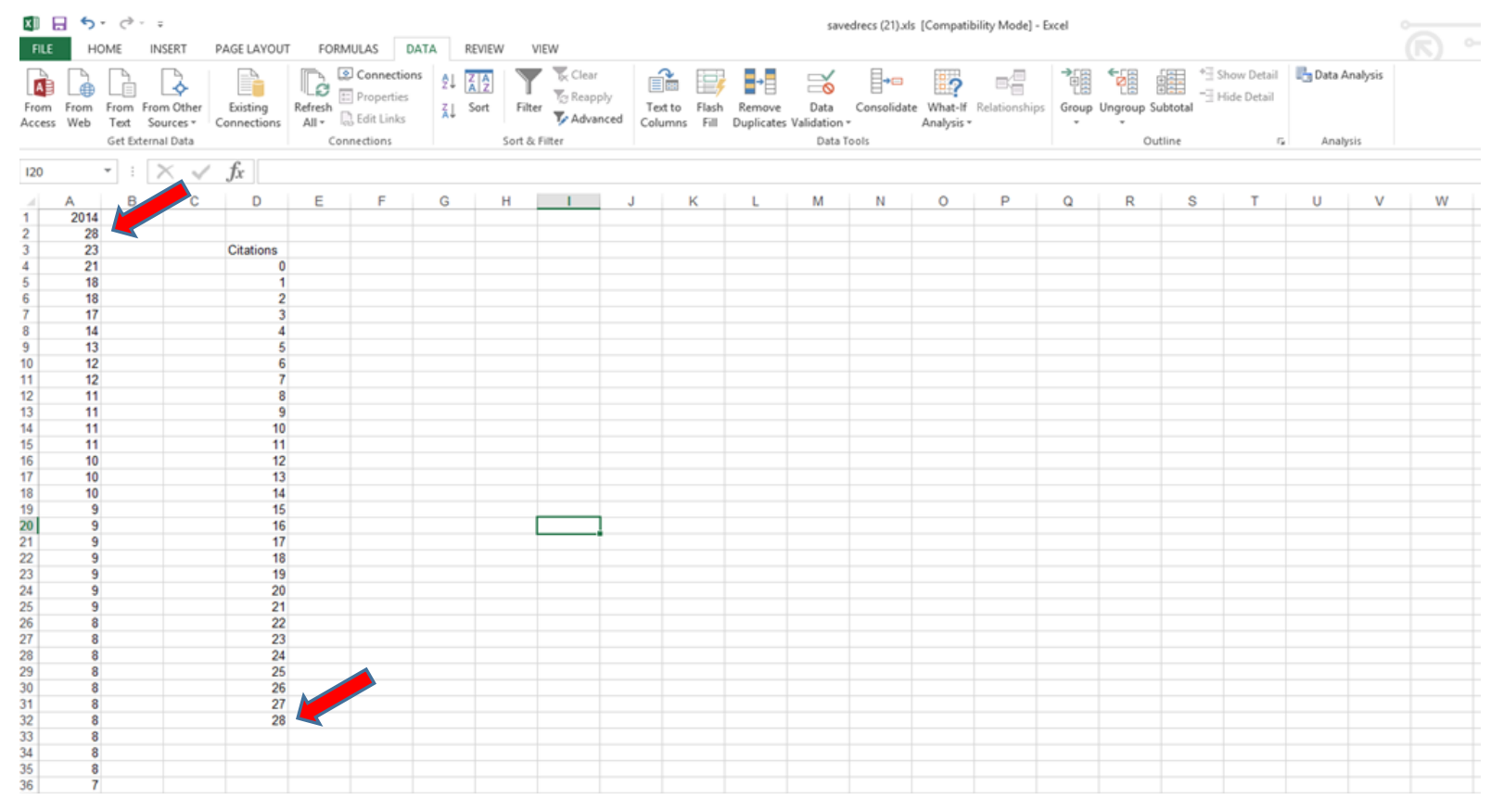

11. Enter the formula $=\operatorname{COUNTIF}(A: A, D 4)$ into the cell next to the 0 citations (where $A$ is the column containing the citations, and D4 is the cell indicating zero citations - see below).

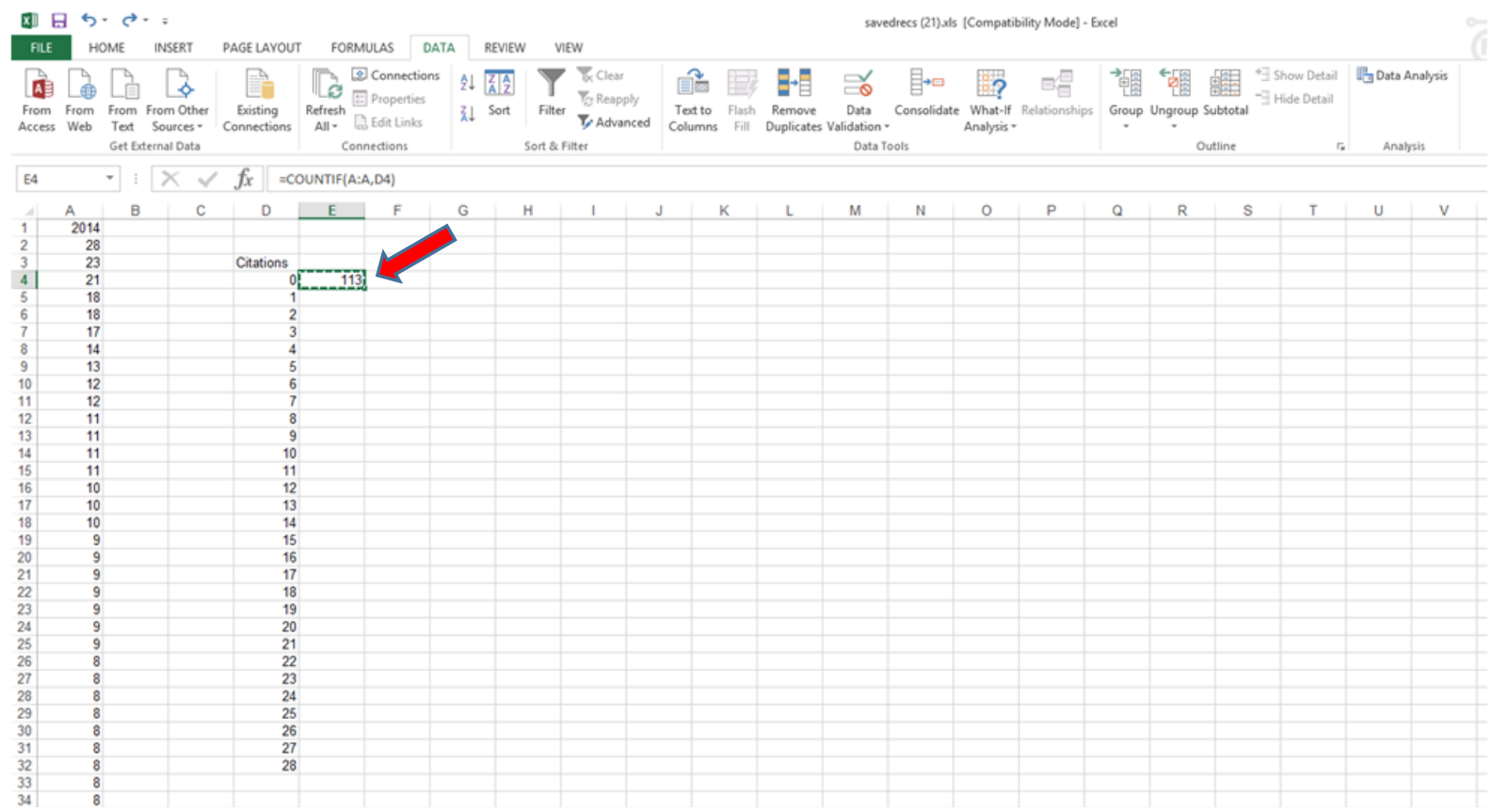


12. Copy and paste this formula into the remaining cells in the Citations column. This generates the data for the frequency distribution.

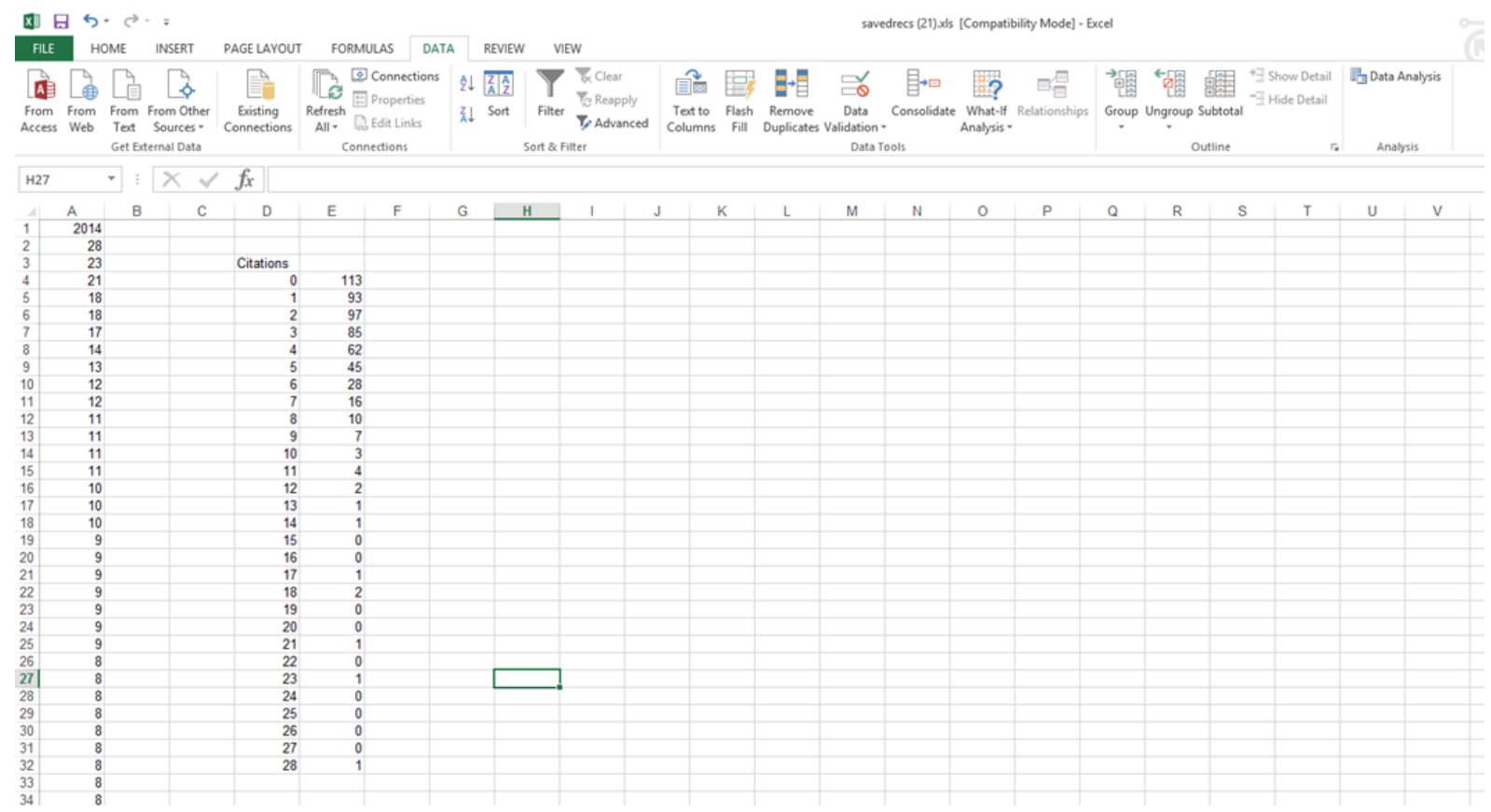

13. If you wish to determine the median, use Excel's MEDIAN function on column A (excluding the 2014 label).

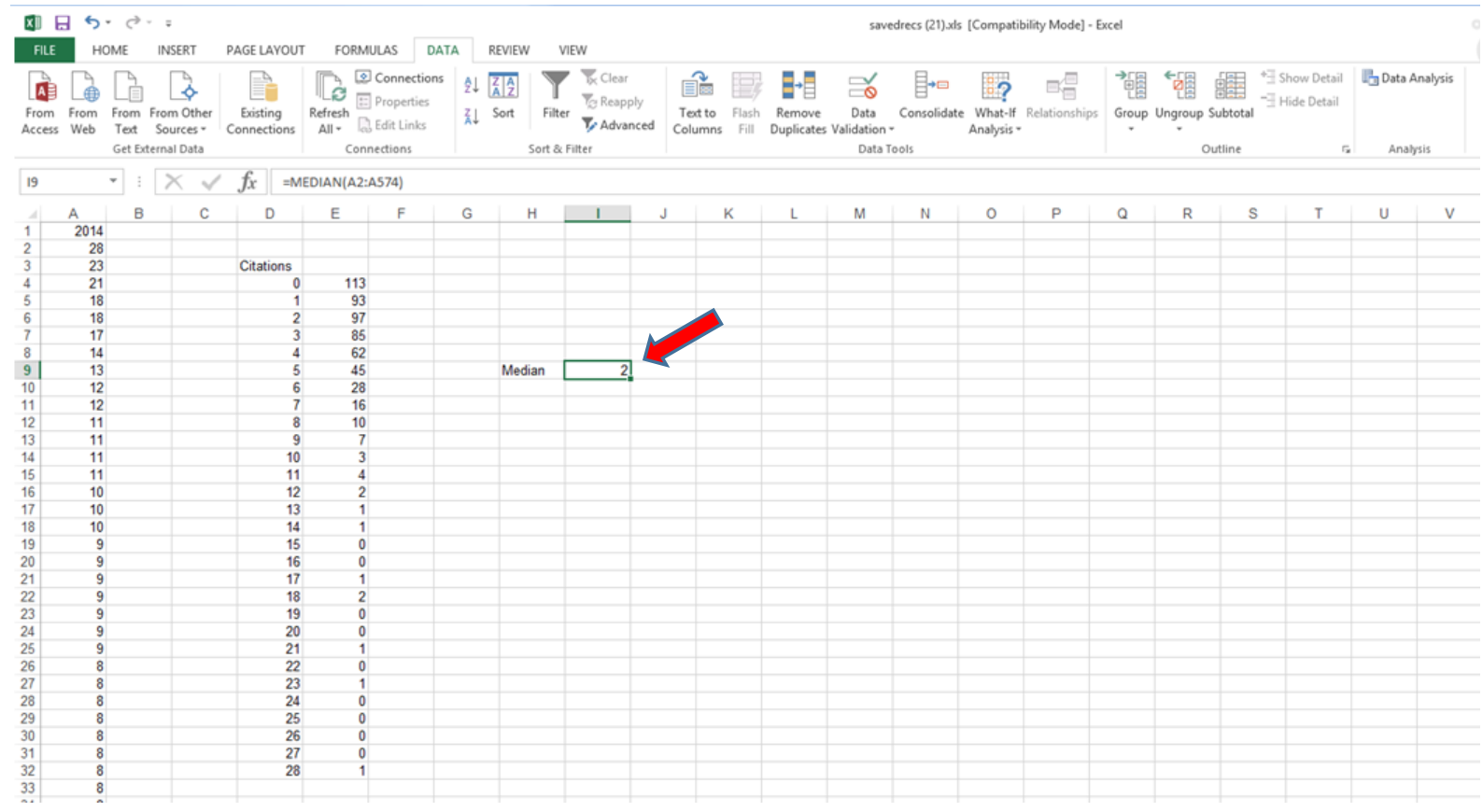


bioRxiv preprint doi: https://doi.org/10.1101/062109. The copyright holder for this preprint (which was not peer-reviewed) is the author/funder. It is made available under a CC-BY 4.0 International license.

Larivière et al. (2016) - Publication of Journal Citations

14. Then make a bar chart with the "Citations" field as the x-axis and the frequency counts as the $y$-axis. If desired, add vertical lines to indicate the JIF and the Median.

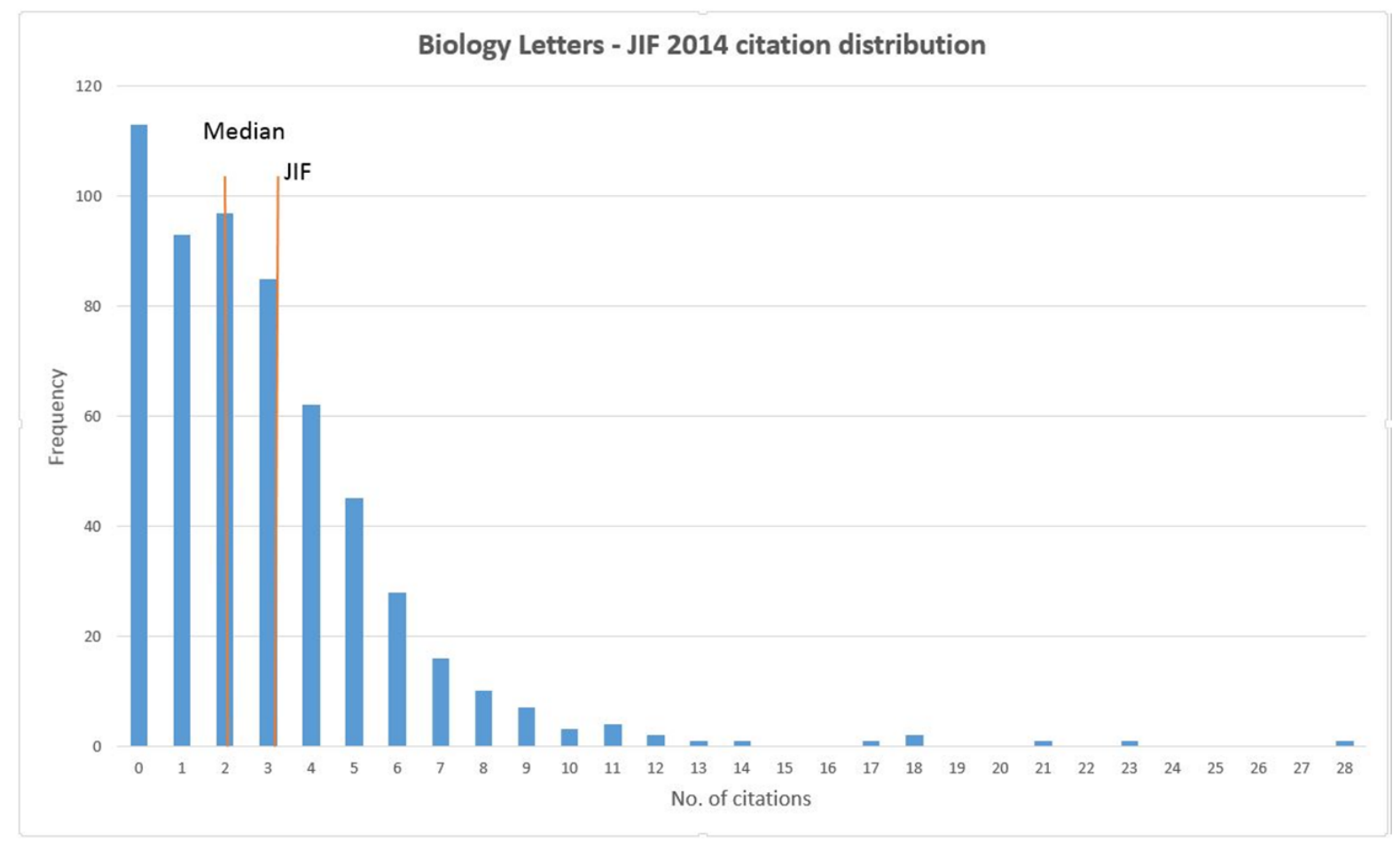


Appendix 2 - Method for generating the journal citation distribution graph from Scopus ${ }^{\mathrm{TM}}$ (2014 Impact Factor set)

The example given below is for generating distributions over the two-year window (2012-2013) that is used in calculation of the 2014 Journal Impact Factor. For later years, the two-year window should be adjusted accordingly.

1. In Scopus ${ }^{\mathrm{TM}}$, search for the journal using the 'Source Title' field (or print ISSN or online ISSN) and select the date range 2012-2013. Journal editors should check the resulting hit-list against the journal's own records as tests showed that the numbers of articles returned may differ depending on which field is used for the search. Users without access to internal records can check article counts via tables of contents.

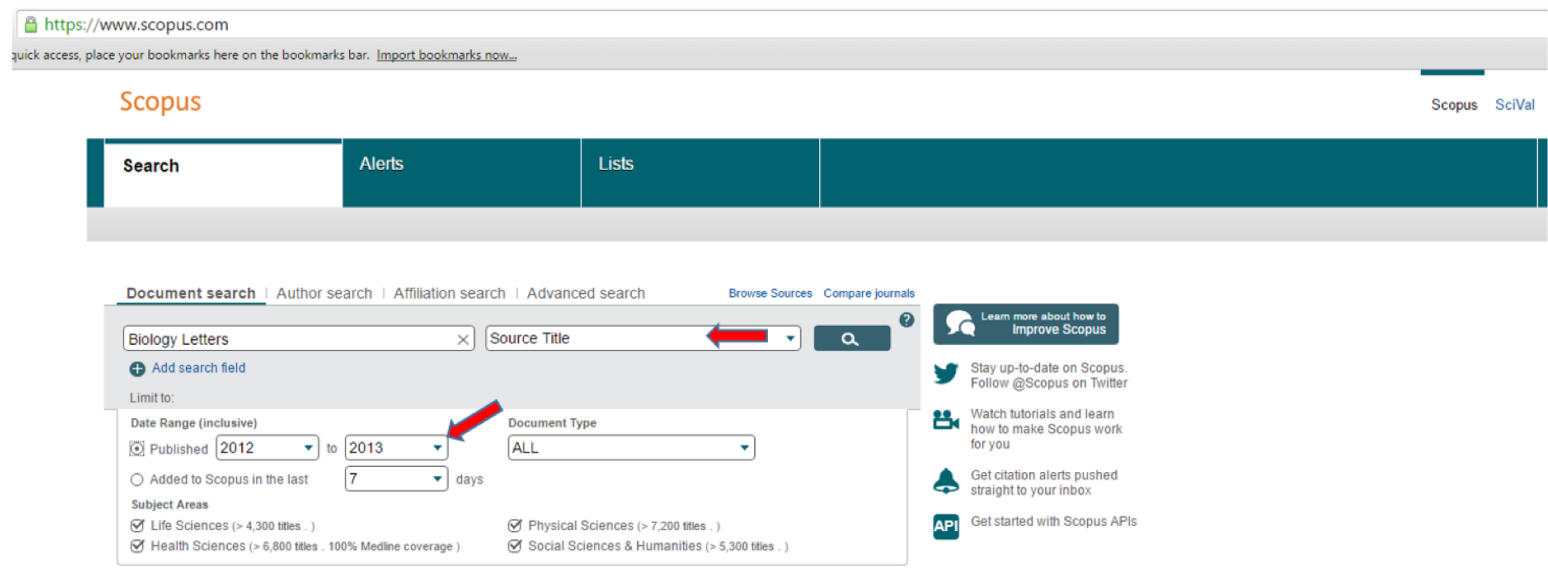

2. "Select all" from the resulting hit-list. (To match as closely as possible the distributions shown in the analyses in this paper, limit the document types in the search to 'Articles' and 'Reviews' using the buttons on the left hand side of the screen.)

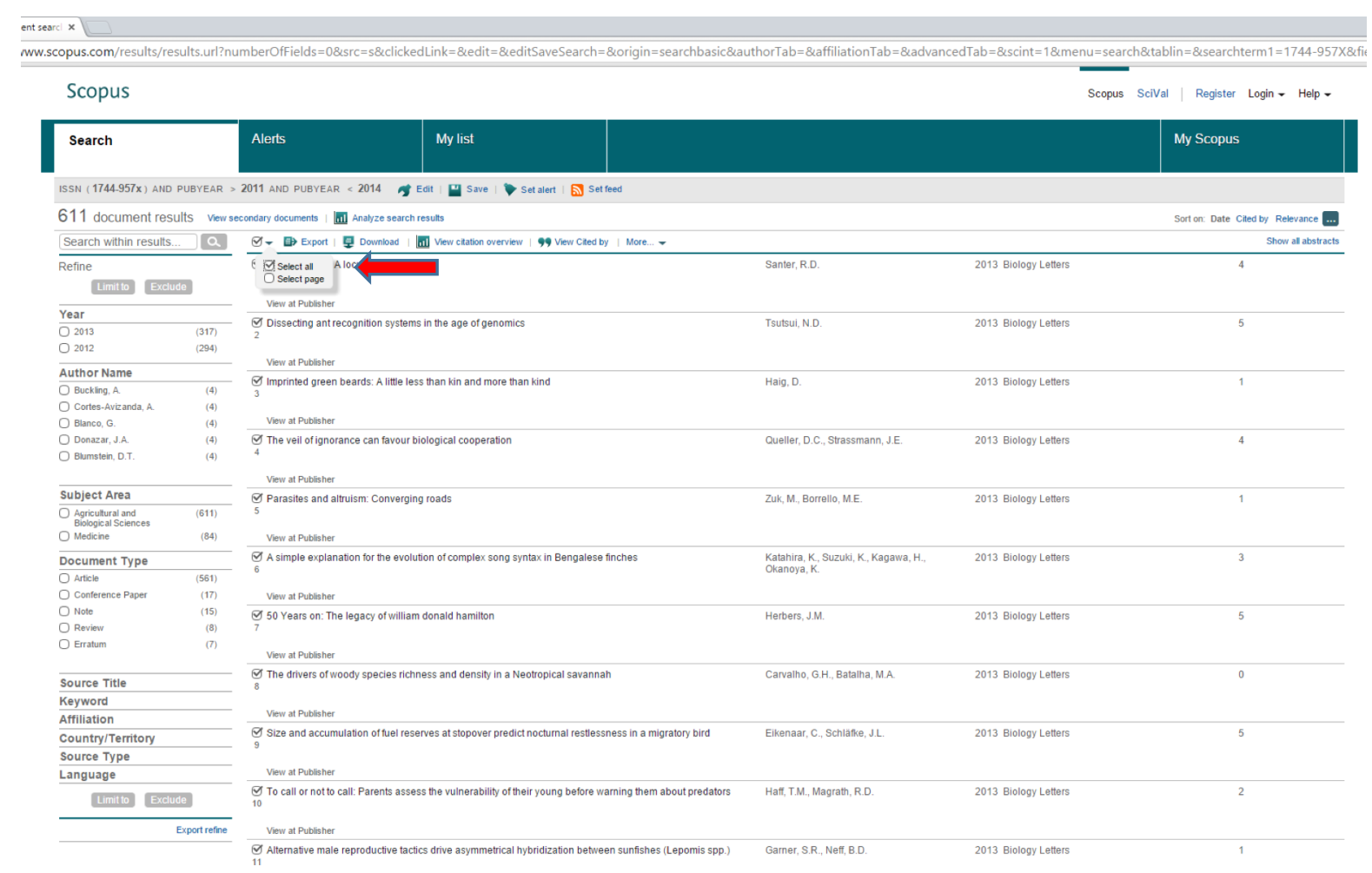


bioRxiv preprint doi: https://doi.org/10.1101/062109. The copyright holder for this preprint (which was not peer-reviewed) is the author/funder. It is made available under a CC-BY 4.0 International license.

Larivière et al. (2016) - Publication of Journal Citations

\section{Click "view citation overview".}

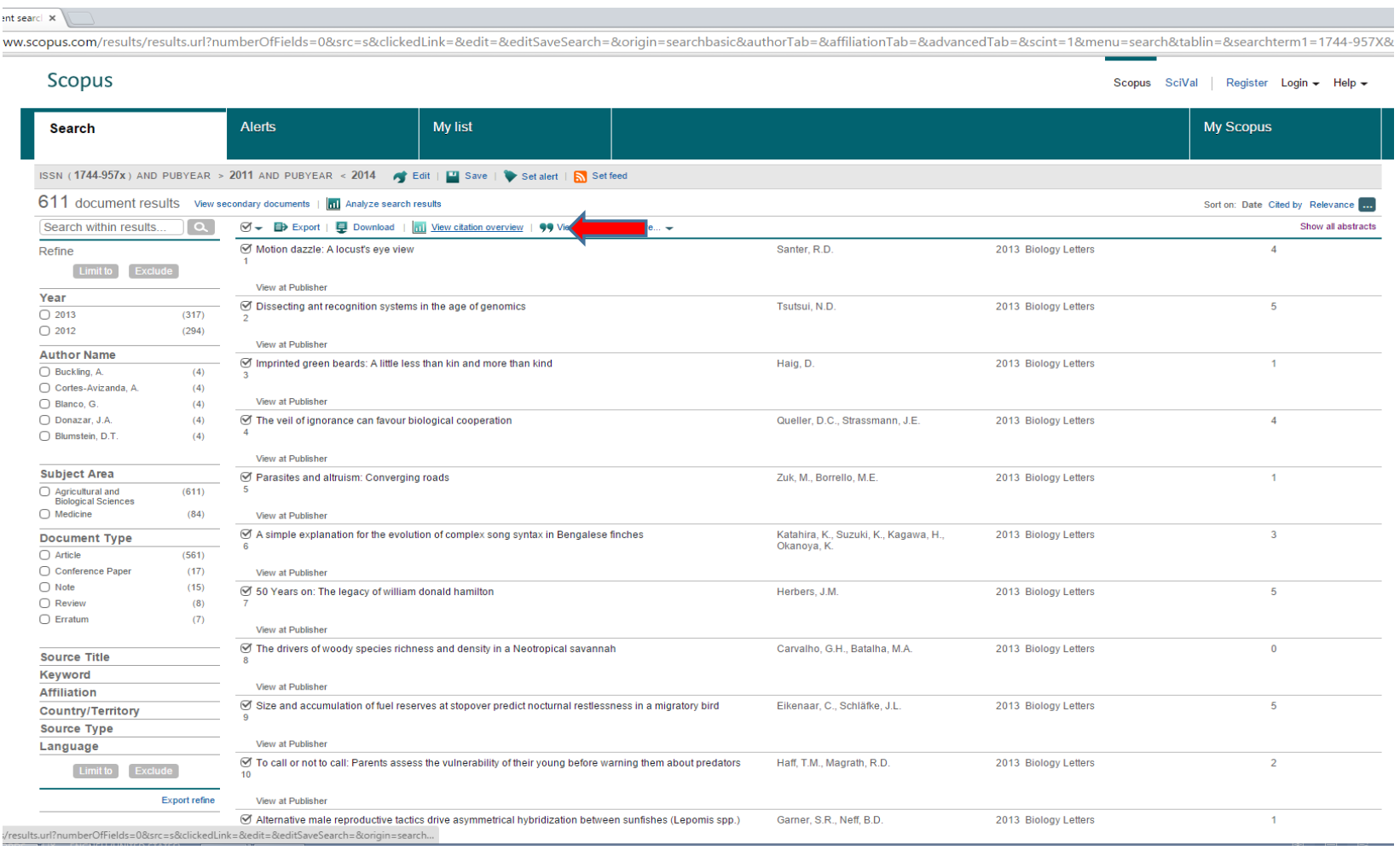

\section{The Citation Overview will look something like this:}

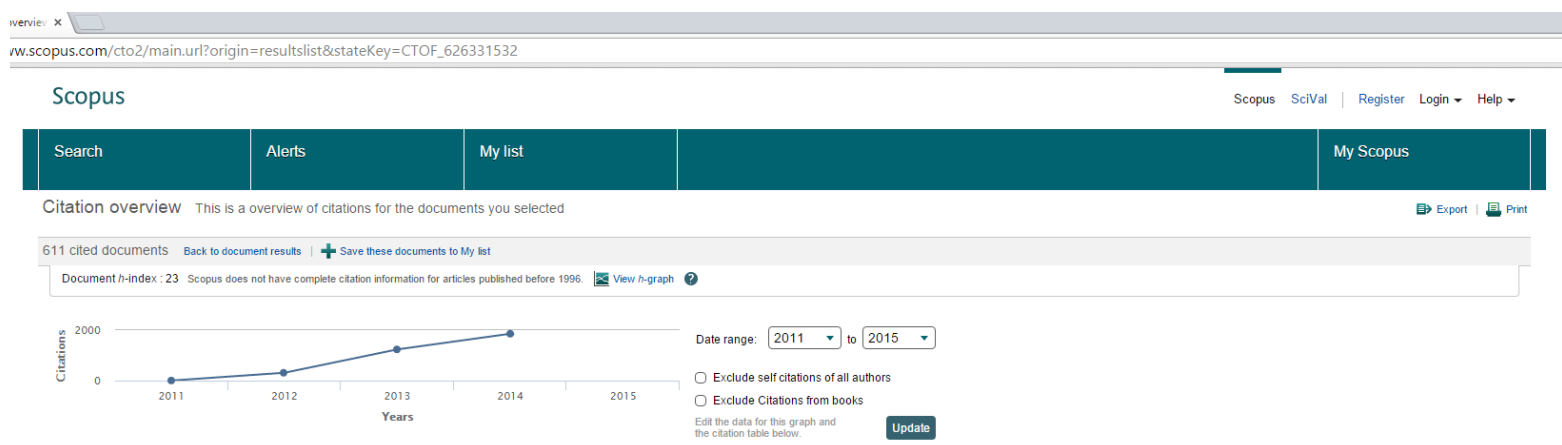

\begin{tabular}{|c|c|c|c|c|c|c|c|c|c|c|}
\hline \multirow{3}{*}{$\begin{array}{l}\text { Documents } \\
\text { Sort on: Date (newest) Citation count (descencing) }\end{array}$} & \multicolumn{8}{|c|}{ Citations } & \multirow[b]{2}{*}{$=2015$} & \multirow[b]{2}{*}{ Total } \\
\hline & & $<2011$ & 2011 & 2012 & 2013 & 2014 & 2015 & Subtotal & & \\
\hline & Total & 6 & 4 & 312 & ${ }_{1237}$ & 1857 & 1426 & 4836 & 9 & 4851 \\
\hline 1 Motion dazzle: A locust's eye vievr & 2013 & & & & & 3 & 1 & 4 & & ${ }^{4}$ \\
\hline 2 Dissecting antrecognntion systems in the age of genomics & 2013 & & & & 1 & 2 & 2 & 5 & & 5 \\
\hline 3 Imprinited green beards: A little less than kin and more than.... & 2013 & & & & 1 & & & 1 & & 1 \\
\hline 4 The veli ofignorance can favour biological cooperation & 2013 & & & & 1 & 1 & 2 & 4 & & 4 \\
\hline 5 Perasites and altruism: Converging roads & 2013 & & & & 1 & & & 1 & & 1 \\
\hline 6 A simple explanation ior the evolution of complex song synta. & 2013 & & & & & 2 & 1 & 3 & & 3 \\
\hline 750 Years on: The legacy or willam donald hamilton & 2013 & & & & & 3 & 2 & 5 & & 5 \\
\hline 8 The divers ofwoody species richness and densily in a Neott... & 2013 & & & & & & & 0 & & 0 \\
\hline 9 Size and accumulation offuel reserves at stopover predict n. & 2013 & & & & & 3 & 2 & 5 & & 5 \\
\hline 10 To call or not to call: Parents assess the vulnerability or... & 2013 & & & & & 1 & 1 & 2 & & 2 \\
\hline 11 Allernative male reproductive tactics drive asymmetrical hyb... & 2013 & & & & & 1 & & 1 & & 1 \\
\hline 12 Hamiltonian inclusive fitness:A fitter finess concept & 2013 & & & & 1 & & & 1 & & 1 \\
\hline 13 Brains and the city in passerine birds. Re-Analysis and cont.. & 2013 & & & & & & & 0 & & 0 \\
\hline 14 Sex difierences in the proiection of host immune systems by ... & 2013 & & & & & & & 0 & & 0 \\
\hline 15 lanoring discards biases the assessment of fisheries ' ccoloo... & 2013 & & & & & 1 & 1 & 2 & & 2 \\
\hline 16 Social forrgers adopta riskier foraging mode in the cente ... & 2013 & & & & & 2 & & 2 & & 2 \\
\hline
\end{tabular}


bioRxiv preprint doi: https://doi.org/10.1101/062109. The copyright holder for this preprint (which was not peer-reviewed) is the author/funder. It is made available under a CC-BY 4.0 International license.

Larivière et al. (2016) - Publication of Journal Citations

5. Select the date range 2014 (to get only citations in 2014) and click "update".

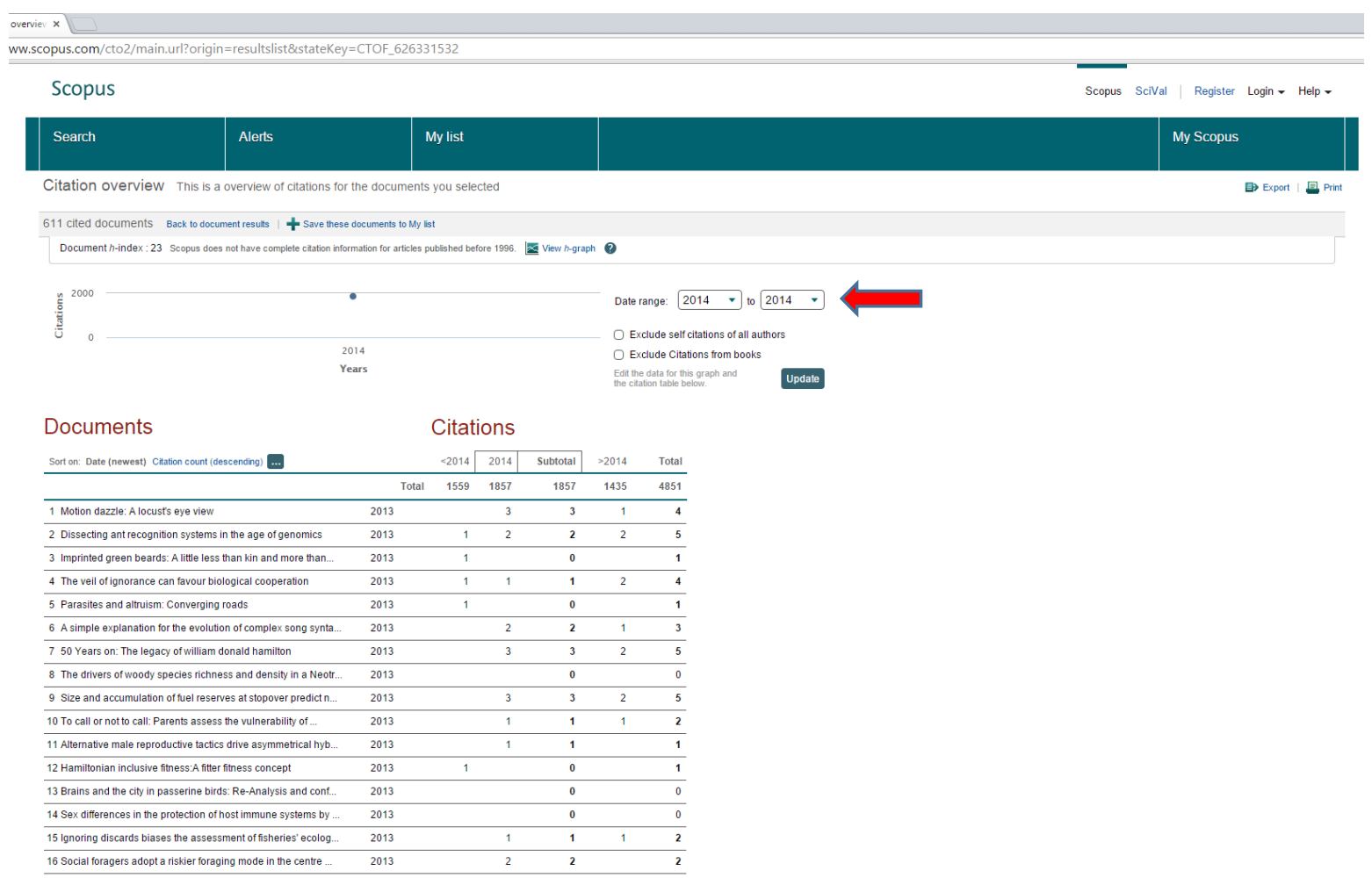

\section{Then click "Export".}

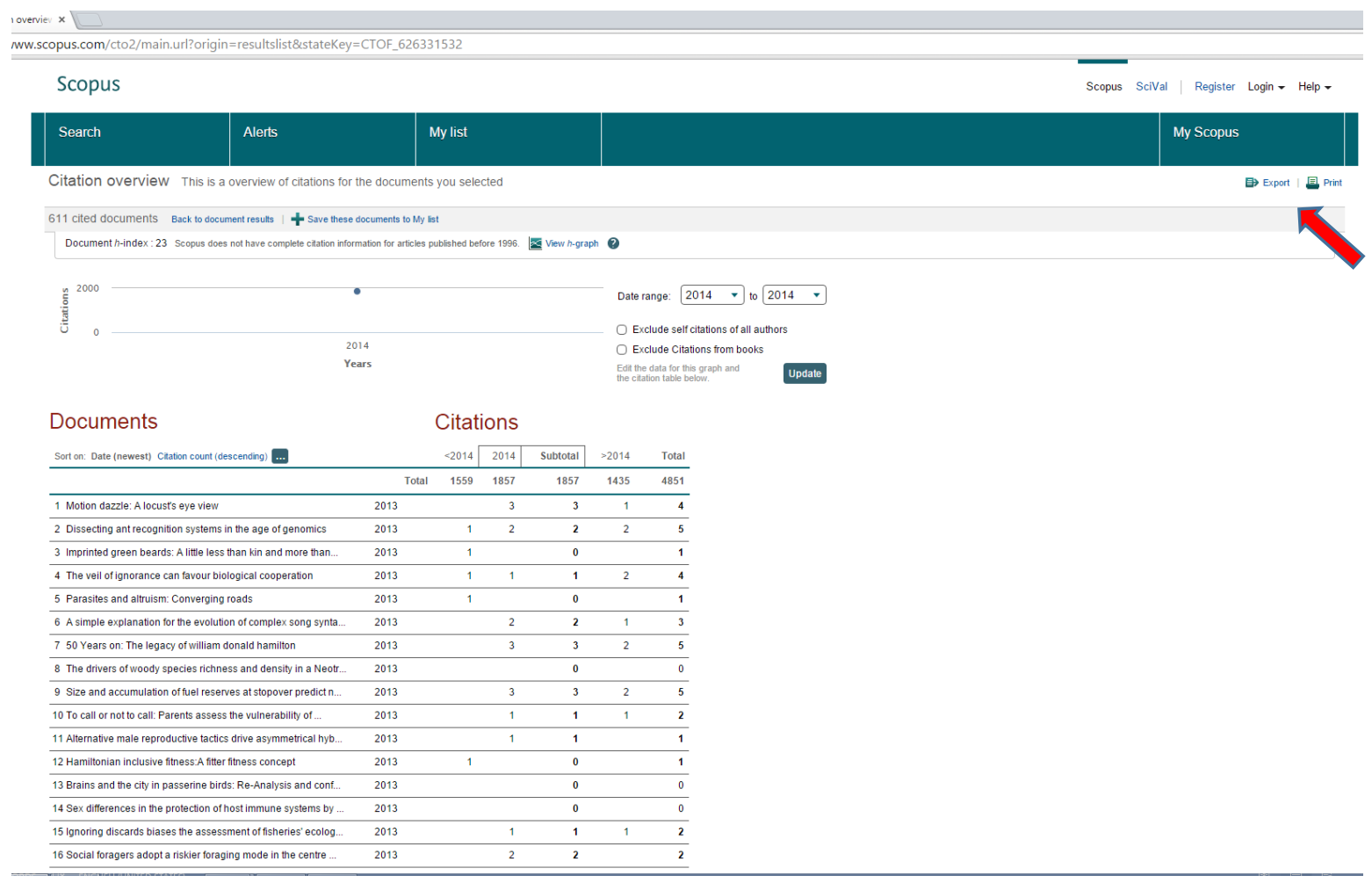


bioRxiv preprint doi: https://doi.org/10.1101/062109. The copyright holder for this preprint (which was not peer-reviewed) is the author/funder. It is made available under a CC-BY 4.0 International license.

Larivière et al. (2016) - Publication of Journal Citations

\section{This will download a CSV (comma-separated values) file. Open it in Excel.}

国早穴-

CTOExport (2).csv - Excel

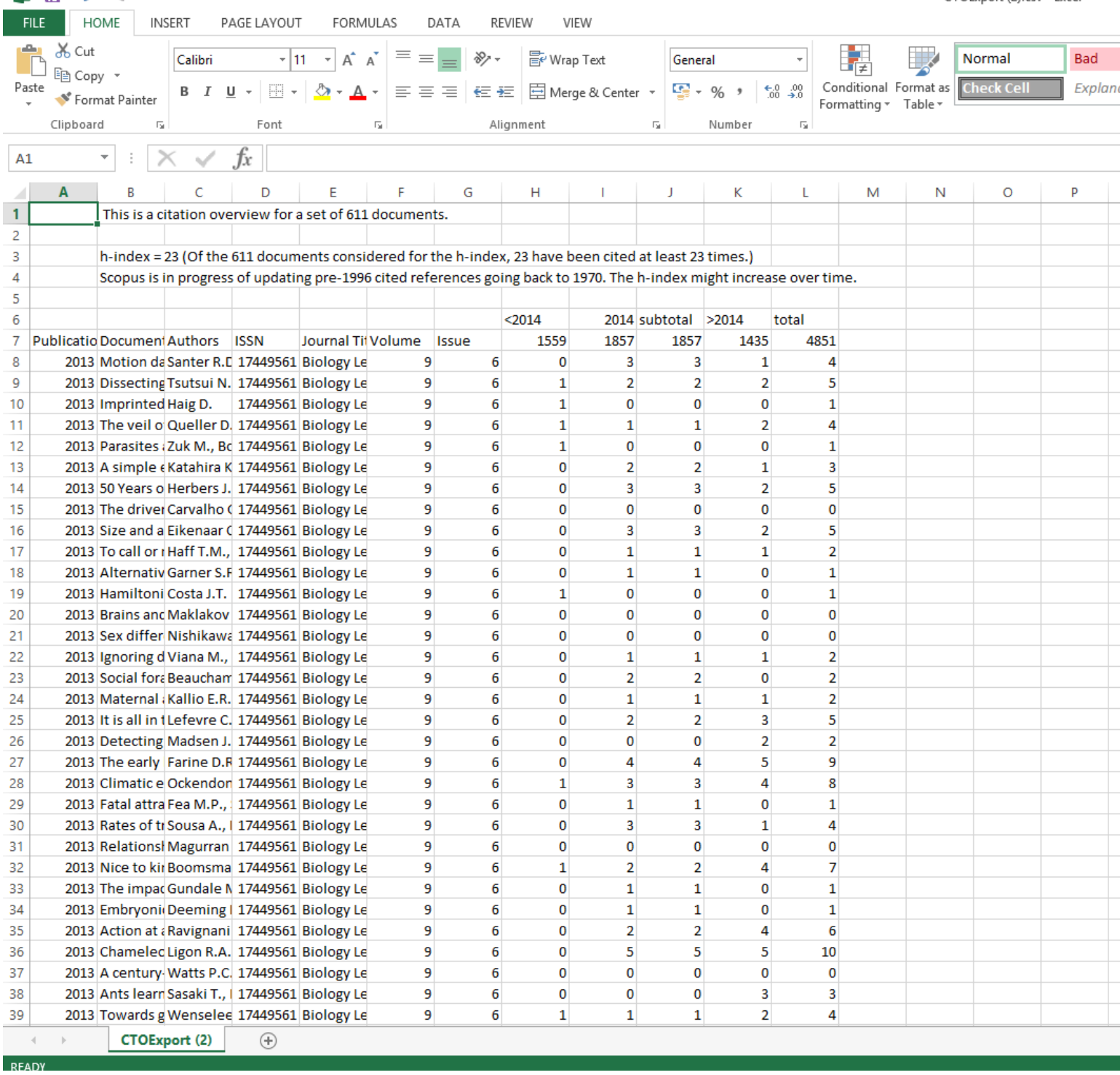


bioRxiv preprint doi: https://doi.org/10.1101/062109. The copyright holder for this preprint (which was not peer-reviewed) is the author/funder. It is made available under a CC-BY 4.0 International license.

Larivière et al. (2016) - Publication of Journal Citations

\section{Scopus ${ }^{\mathrm{TM}}$ may contain duplicate records for the same paper that have both accumulated citations. To resolve this, sort the records on the title column (A-Z) to make it easy to identify duplicates. For each pair, delete one, but make sure to add its citation count (e.g. in the 2014 column) to the remaining one to produce the correct total.}

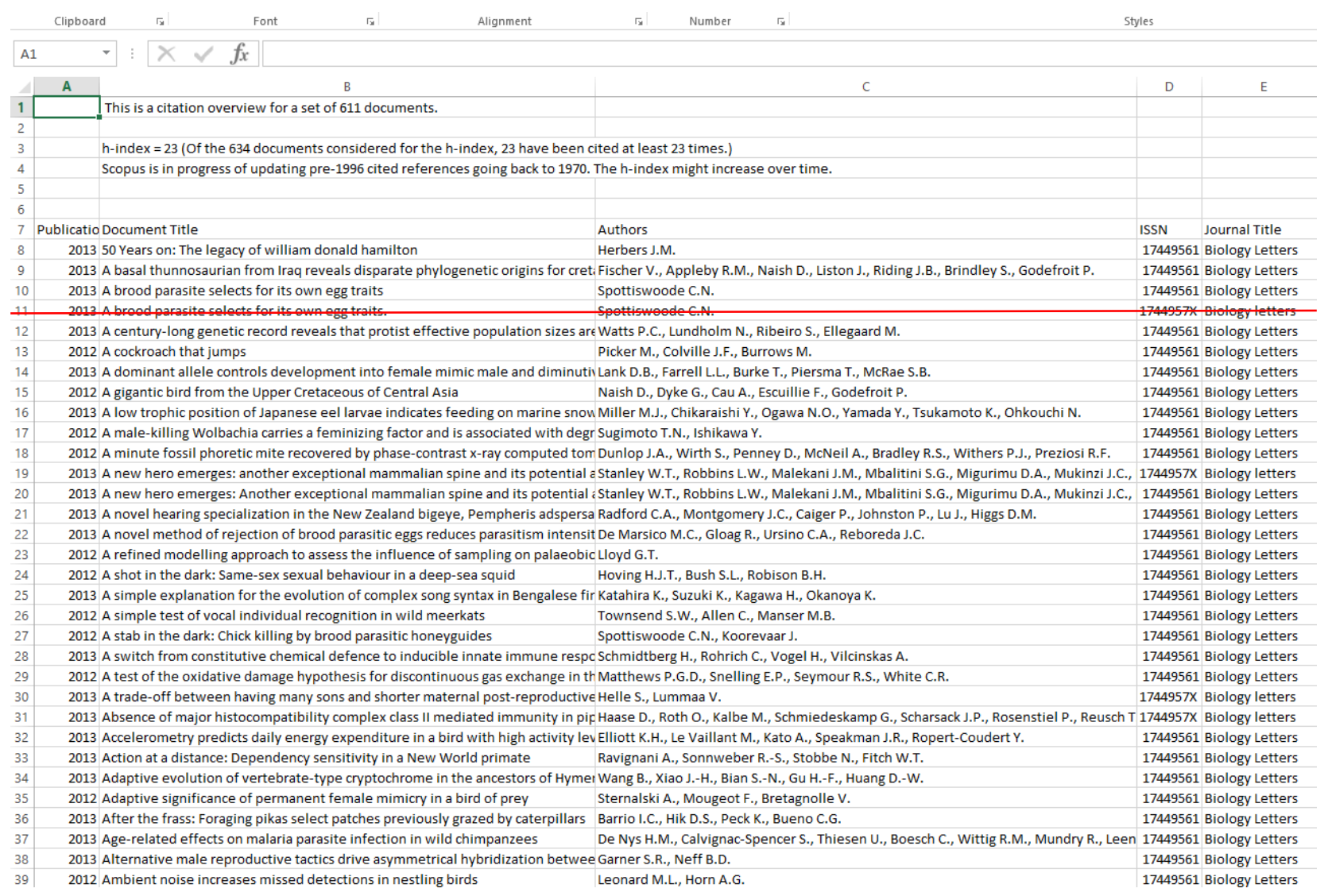


bioRxiv preprint doi: https://doi.org/10.1101/062109. The copyright holder for this preprint (which was not peer-reviewed) is the author/funder. It is made available under a CC-BY 4.0 International license.

Larivière et al. (2016) - Publication of Journal Citations

\section{After de-duplication of the data, select the column for the citations received in 2014; (the other columns can be deleted).}

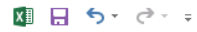

FILE HOME INSERT PAgelayout Formulas DATA REVIEW VIEW

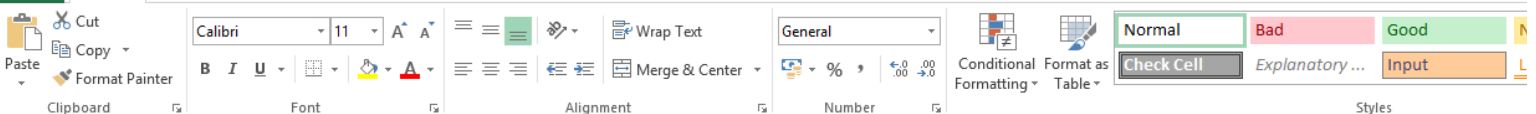

\begin{tabular}{|c|c|c|c|c|c|c|c|c|c|c|c|c|c|c|c|c|c|c|}
\hline & A & $c$ & $D$ & $E$ & $F$ & $G$ & $\mathrm{H}$ & 1 & & K & L & $\mathrm{M}$ & $\mathrm{N}$ & 0 & P & $\mathrm{Q}$ & R & $\mathrm{s}$ \\
\hline $\begin{array}{l}1 \\
2\end{array}$ & & \multicolumn{17}{|c|}{ This is a citation overview for a set of 611 documents. } \\
\hline 3 & & \multirow{2}{*}{\multicolumn{6}{|c|}{$\begin{array}{l}\text { h-index }=23 \text { (Of the } 611 \text { documents considered for the } h \text {-index, } 23 \text { have } \\
\text { Scopus is in progress of updating pre-1996 cited references going back to }\end{array}$}} & been cited & at least 23 & B times.) & & & & & & & & \\
\hline 4 & & & & & & & & 1970. The & \multicolumn{4}{|c|}{ h-index might increase over time. } & & & & & & \\
\hline 5 & & & & & & & & & & & & & & & & & & \\
\hline 6 & & & & & & & $<2014$ & 2014 & subtotal & $>2014$ & total & & & & & & & \\
\hline 9 & 2013 & Dissecting Tsutsui N. & $17449561 \mathrm{E}$ & Biology Le & 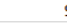 & 9 & 6 & 2 & 2 & 2 & 5 & & & & & & & \\
\hline 10 & 2013 I & Imprinted Haig D. & 17449561 & Biology LE & & 9 & 6 & 0 & 0 & 0 & 1 & & & & & & & \\
\hline 11 & $2013 \mathrm{~T}$ & The veil o Queller $\mathrm{D}$. & $17449561 \mathrm{E}$ & Biology Le & & 9 & 6 & 1 & 1 & 2 & 4 & & & & & & & \\
\hline 12 & $2013 \mathrm{P}$ & Parasites : Zuk M., BC & 17449561 & Biology Le & ste & 9 & 6 & 0 & 0 & 0 & 1 & & & & & & & \\
\hline 13 & $2013 \mathrm{~A}$ & A simple $\in$ Katahira $K$ & 17449561 & Biology Le & & 9 & 6 & 2 & 2 & 1 & 3 & & & & & & & \\
\hline 14 & 20135 & 50 Years o Herbers J. & 17449561 & Biology Le & s & 9 & 6 & 3 & 3 & 2 & 5 & & & & & & & \\
\hline 15 & $2013 \mathrm{~T}$ & The driver Carvalho $c$ & 17449561 & Biology LE & & 9 & 6 & 0 & 0 & 0 & 0 & & & & & & & \\
\hline 16 & $2013 \mathrm{~s}$ & Size and a Eikenaar C & 17449561 & Biology Le & & 9 & 6 & 3 & 3 & 2 & 5 & & & & & & & \\
\hline 21 & $2013 \mathrm{~s}$ & Sex differ Nishikawe & 17449561 & Biology LE & 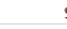 & 9 & 6 & 0 & 0 & 0 & 0 & & & & & & & \\
\hline 22 & 2013 I & Ignoring d Viana M., & 17449561 & Biology Le & & 9 & 6 & 1 & 1 & 1 & 2 & & & & & & & \\
\hline 23 & $2013 \mathrm{~s}$ & Social for Beauchar & 17449561 & Biology Le & & 9 & 6 & 2 & 2 & 0 & 2 & & & & & & & \\
\hline 24 & $2013 n$ & Maternal : Kallio E.R. & 17449561 & Biology LE & & 9 & 6 & 1 & 1 & 1 & 2 & & & & & & & \\
\hline 25 & 2013 I & It is all in 1 Lefevre C. & $17449561 \mathrm{E}$ & Biology LE & & 9 & 6 & 2 & 2 & 3 & 5 & & & & & & & \\
\hline 26 & 2013 & Detecting Madsen J. & 17449561 & Biology LE & & 9 & 6 & 0 & 0 & 2 & 2 & & & & & & & \\
\hline 27 & $2013 \mathrm{~T}$ & The early Farine D.F & 17449561 & Biology LE & & 9 & 6 & 4 & 4 & 5 & 9 & & & & & & & \\
\hline 28 & 2013 & Climatic e Ockendor & 17449561 & Biology LE & & 9 & 6 & 3 & 3 & 4 & 8 & & & & & & & \\
\hline 29 & $2013 \mathrm{~F}$ & Fatal attra Fea M.P., & 17449561 & Biology LE & ster 0 & 9 & 6 & 1 & 1 & 0 & 1 & & & & & & & \\
\hline 30 & $2013 \mathrm{~F}$ & Rates of tı Sousa A., I & 17449561 & Biology Le & & 9 & 6 & 3 & 3 & 1 & 4 & & & & & & & \\
\hline 31 & $2013 \mathrm{~F}$ & Relationsi Magurran & 17449561 & Biology LE & & 9 & 6 & 0 & 0 & 0 & 0 & & & & & & & \\
\hline 32 & $2013 \mathrm{~N}$ & Nice to kit Boomsma & 17449561 & Biology LE & s & 9 & 6 & 2 & 2 & 4 & 7 & & & & & & & \\
\hline 33 & $2013 \mathrm{~T}$ & The impac Gundale A & 17449561 & Biology LE & & 9 & 6 & 1 & 1 & 0 & 1 & & & & & & & \\
\hline 34 & 2013 & Embryoni, Deeming I & 17449561 & Biology LE & 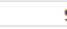 & 9 & 6 & 1 & 1 & 0 & 1 & & & & & & & \\
\hline 35 & $2013 \mathrm{~A}$ & Action at i Ravignani & 17449561 & Biology LE & & 9 & 6 & 2 & 2 & 4 & 6 & & & & & & & \\
\hline 36 & 2013 & Chamelec Ligon R.A. & 17449561 & Biology Le & & 9 & 6 & 5 & 5 & 5 & 10 & & & & & & & \\
\hline 39 & $2013 \mathrm{~T}$ & Towards g Wensele $\epsilon$ & 17449561 & Biology Le & & 9 & 6 & 1 & 1 & 2 & 4 & & & & & & & \\
\hline
\end{tabular}


10. Sort the column into descending order - make sure to omit the row labels.

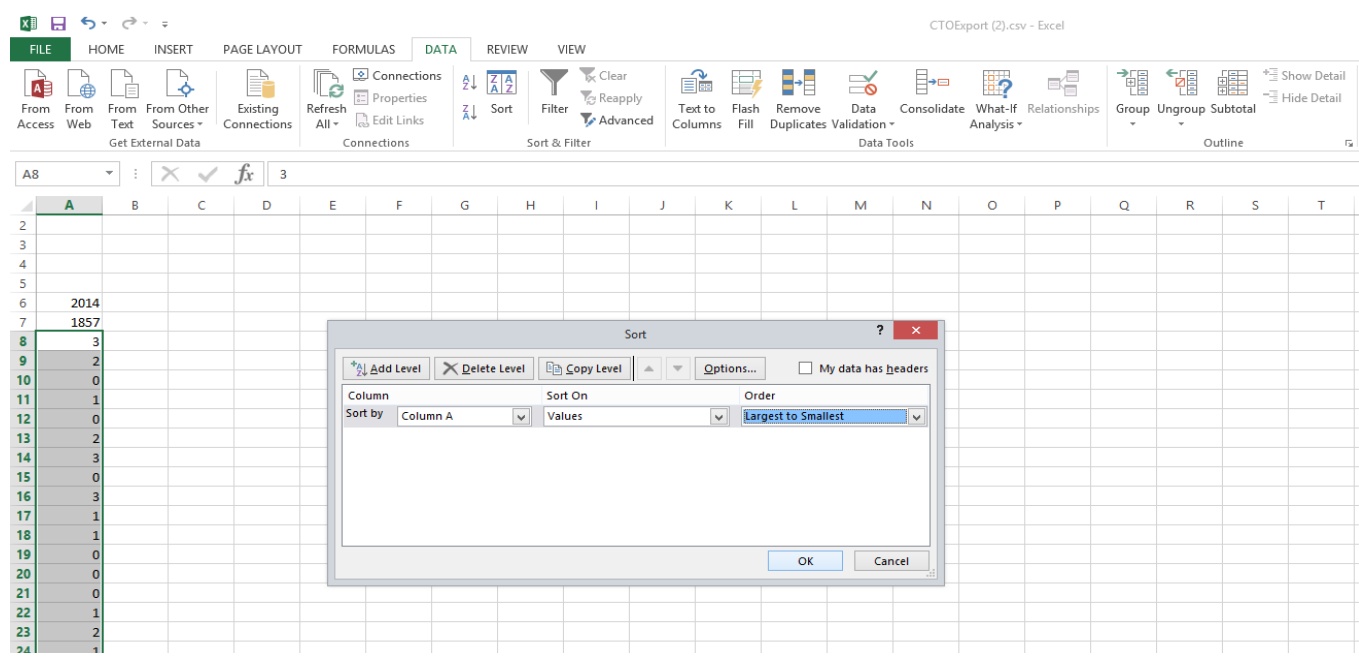

11. Note the maximum citation $(x)$ count and create a new column containing 0 to $X$ called "Citations". In the example shown below, $x=28$.

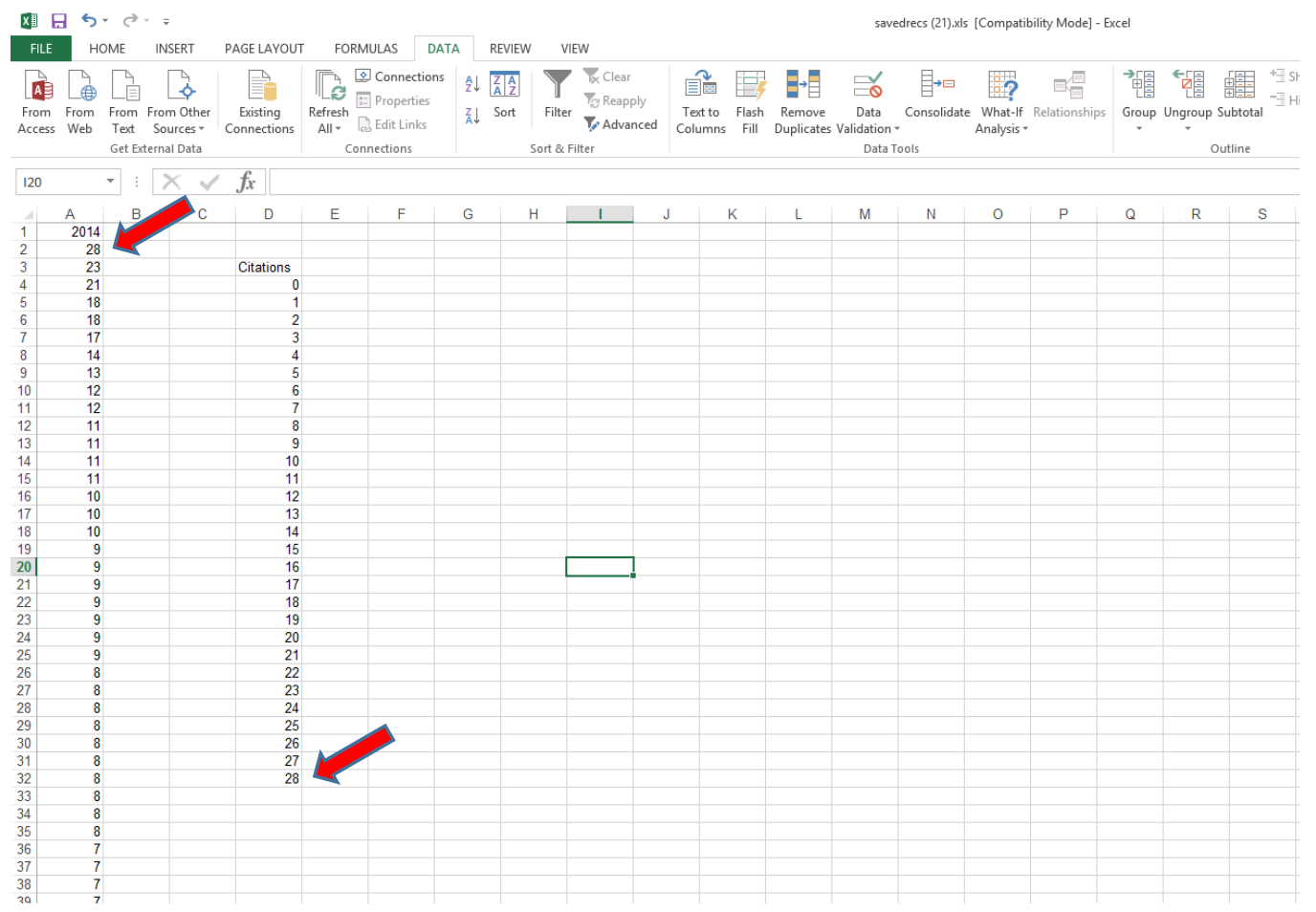


12. Enter the formula $=\operatorname{COUNTIF}(A: A, D 4)$ into the cell next to the 0 citations (where $A$ is the column containing the citations, and D4 is the cell with the zero citation count).

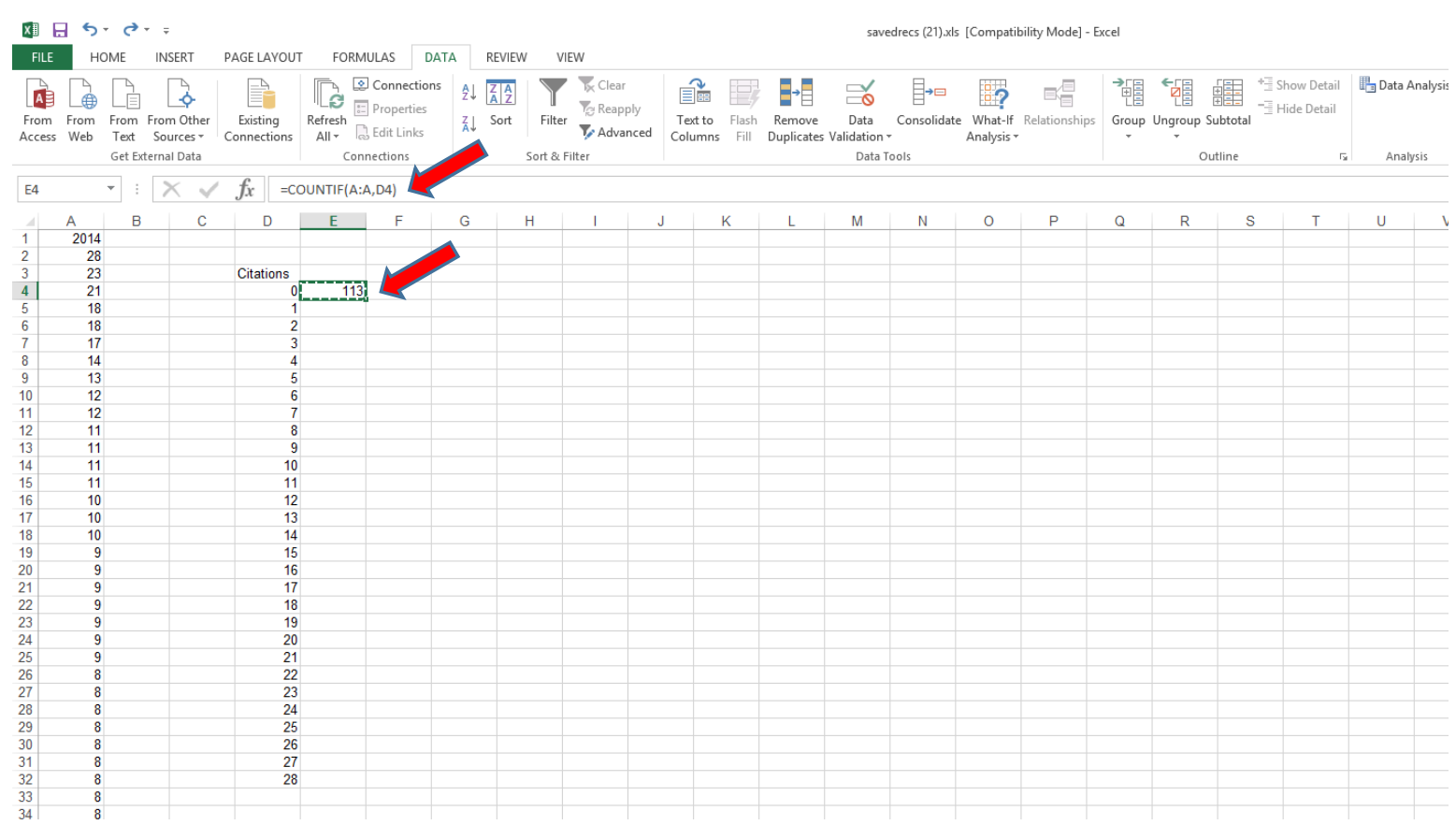

13. Copy and paste this formula into the remaining cells in the Citations column to generate the frequency distribution data.

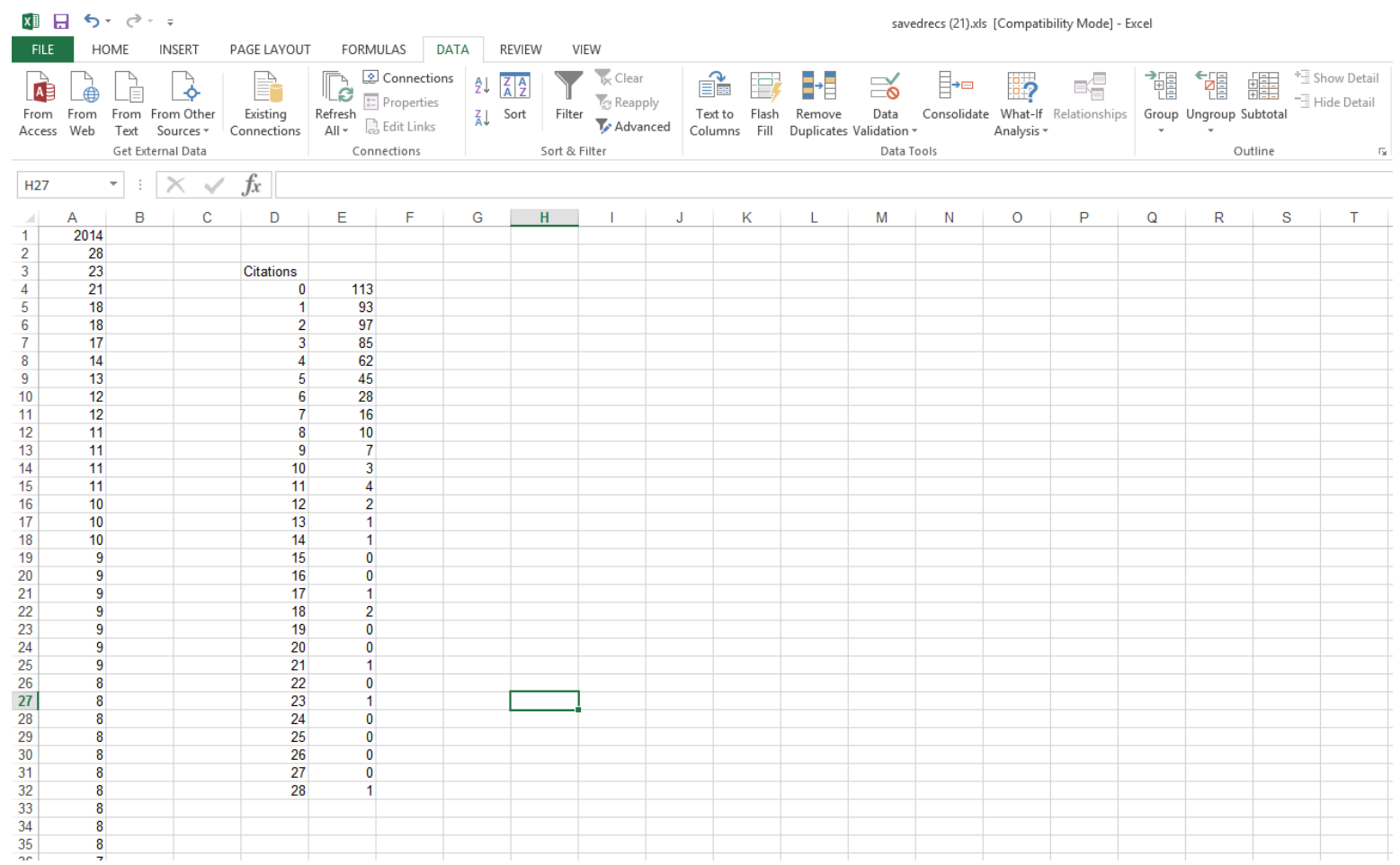


14. If you wish to determine the median, use Excel's MEDIAN function on column A; be careful not to include the '2014' label.

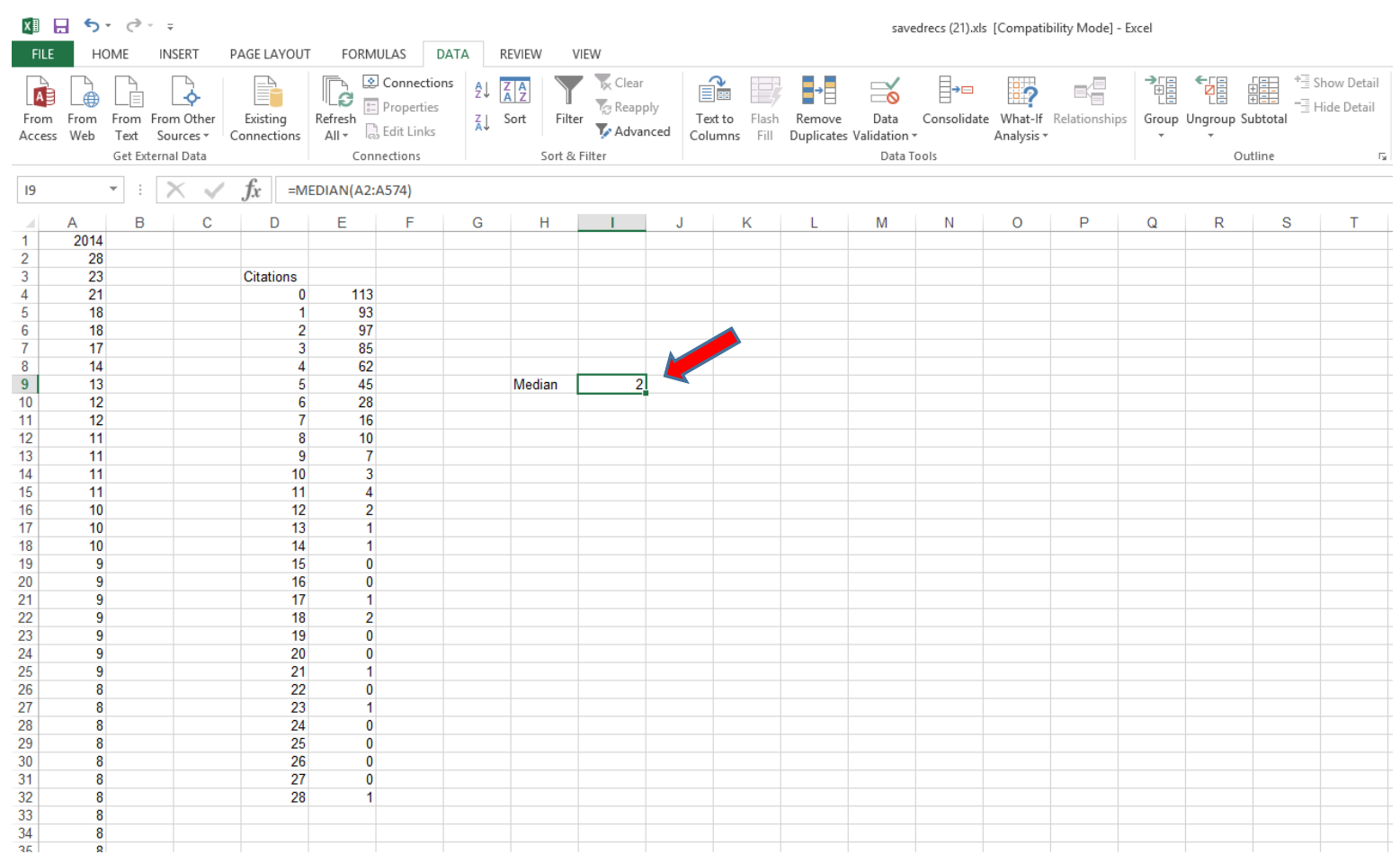

15. Then make a bar chart with the "Citations" field as the x-axis. If desired, add a vertical line to denote the JIF and the Median.

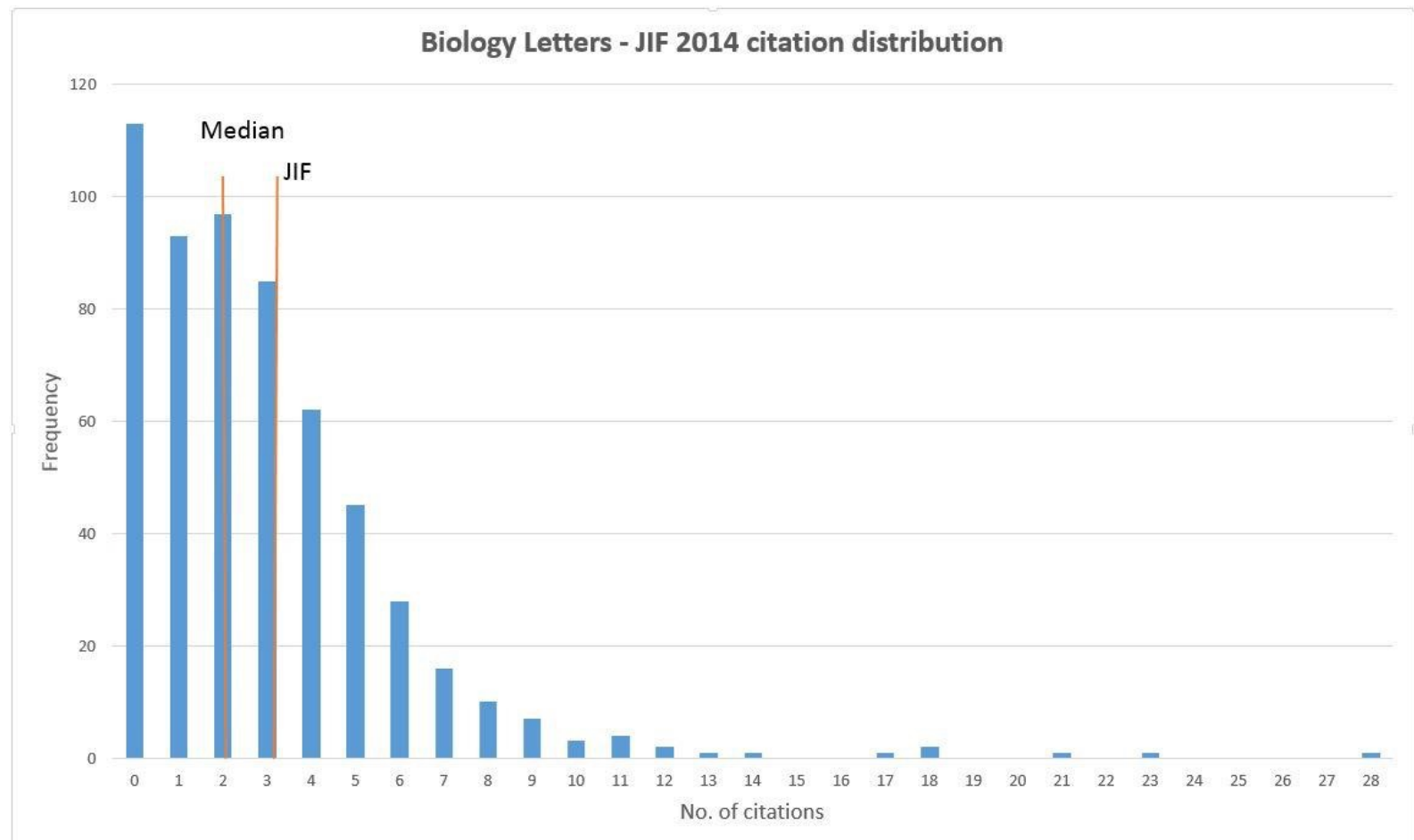

